\title{
Non-synchronous vibration in axial compressors: Lock-in mechanism and semi-analytical model
}

\author{
Sina Stapelfeldt ${ }^{\mathrm{a}, *}$, Christoph Brandstetter ${ }^{\mathrm{b}}$ \\ ${ }^{a}$ Imperial College, Department of Mechanical Engineering, London, United Kingdom \\ ${ }^{b}$ École Centrale de Lyon, Laboratoire de Mecanique des Fluides et d'Acoustique, Lyon, France
}

\begin{abstract}
Non-Synchronous-Vibration (NSV) in high-speed turbomachinery compressors is an aeroelastic phenomenon which can have devastating consequences, including loss of rotor blades. Despite extensive research over the past two decades its underlying mechanisms are not yet understood. This paper aims to explain the physical mechanisms causing NSV in a modern transonic compressor rotor. Referring to previous experimental results and using validated computational fluid dynamics (CFD), a parametric study is performed in order to characterize the aerodynamic disturbance causing NSV, and to understand the lock-in mechanism between the fluid and the structure seen during NSV. The results show that the process is driven by aerodynamics in the tip region. Under highly throttled conditions, the tip leakage flow blocks the passage and causes the disturbance, which is characterised as a vorticity fluctuation, to propagate circumferentially in the leading edge plane. It is found that the propagation speed of the disturbance is determined by the mean flow conditions and only its phase is periodically modulated through interaction with oscillating blades. This is the mechanism facilitating lock-in. Based on these findings a semi-analytic model is developed and calibrated with the numerical results. The model is capable of simulating the lock-in process and correctly predicts unstable vibration modes.
\end{abstract}

Keywords: Axial compressor, non-synchronous vibrations, lock-in

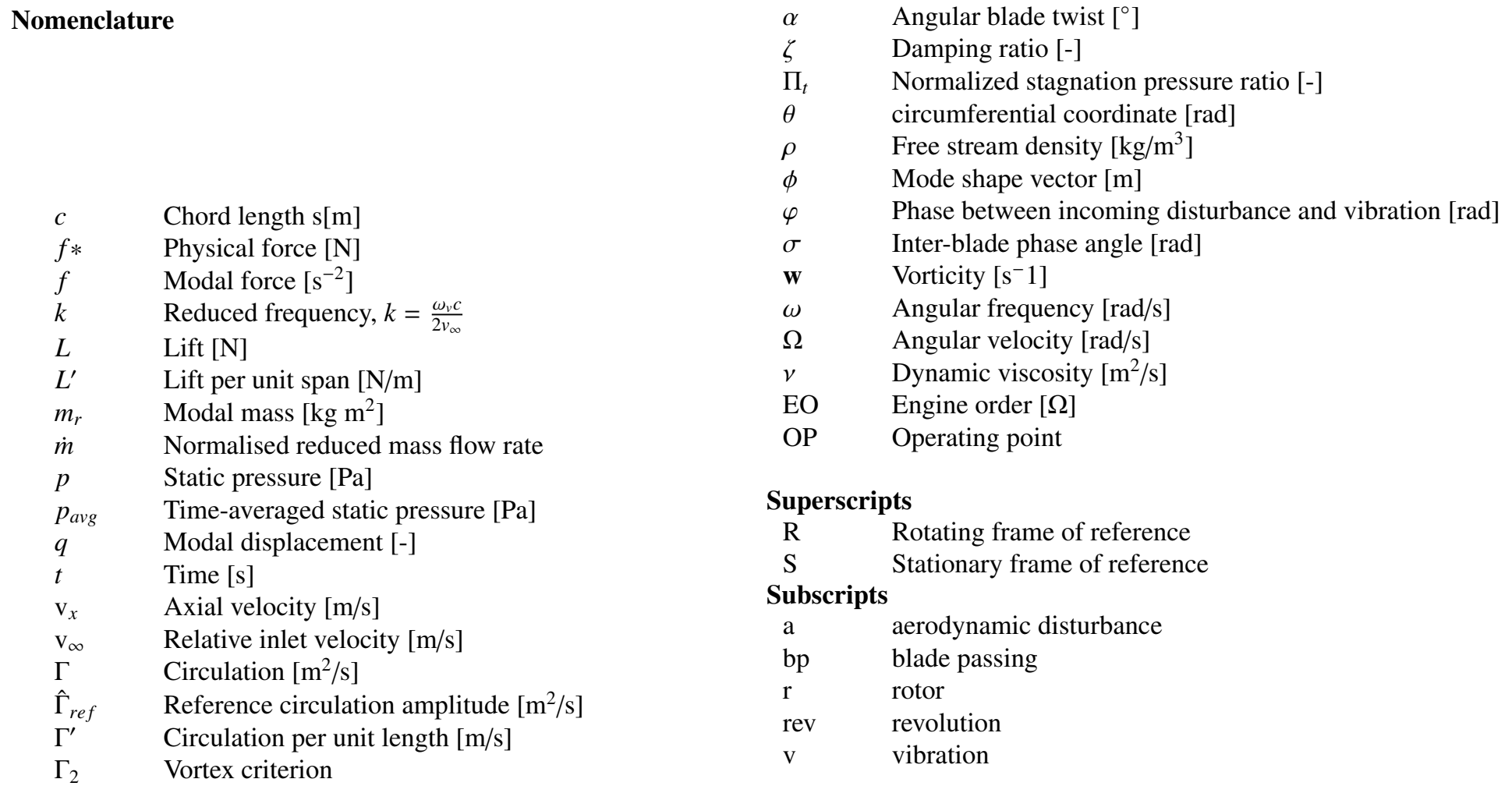

${ }^{*}$ Corresponding author

Email address: s.stapelfeldt@imperial.ac.uk (Sina Stapelfeldt) 


\section{Introduction}

Non-synchronous vibrations in axial compressors were first reported in the 1980s [1] and have since received increasing attention in literature $[2,3,4,5,6]$. The rise in interest is undoubtedly driven by trends in compressor design. Modern designs utilize integrated bladed disks (blisks) with negligible mechanical damping and increased structural coupling through the disk. In addition, highly loaded, low aspect-ratio blades with small profile thickness are sensitive to aerodynamic perturbations, and narrow manufacturing tolerances lead to well-tuned symmetric designs with little aerodynamic damping. All these factors promote coupling between the fluid and structure. It is thus not surprising that non-synchronous vibrations in compressors have recently been reported by all engine manufacturers and are increasingly becoming a design constraint [7, 8, 9, 10].

The term 'non-synchronous vibration' is very general and in theory encompasses any vibration at frequencies not related to the shaft frequency, for example buffeting as well as self-excited vibrations (flutter). However, in recent literature the term has often been used to describe a subset of nonsynchronous vibrations which refuses classification into welldefined types. To align with current literature, the term 'NSV' will be used throughout this paper but a more specific terminology will be proposed towards the end, after the physical mechanisms defining this specific type of NSV investigated have been identified.

According to Kielb et al. [2], the source of NSV is an aerodynamic unsteadiness which locks in with the vibration's natural frequency, with blade dynamics playing a secondary role. It is generally accepted that NSV involves a non-linear aerodynamic behaviour, e.g. the fluid-structure lock-in only takes place once a critical vibration amplitude is reached.

Perhaps due to the general nature of the term 'NSV', the exact cause of it remains unclear. However, there are a few characteristics common to all reported incidences of NSV in axial compressors:

- NSV occurs near the stall boundary but at a stable operating condition.

- Prior to NSV, frequency spectra of unsteady pressure contain broad-band frequencies, which result from multi-wave number disturbances propagating at approximately $50 \%$ of the rotor speed in the direction opposing rotation (in the rotor frame of reference).

- At the onset of NSV, the aerodynamic disturbance locks in with the structural vibration and the broadband spectrum changes to a coherent aerodynamic disturbance with a distinct frequency peak.

These characteristics differentiate NSV from other unsteady aerodynamic and aeroelastic phenomena, such as flutter, buffeting and rotating stall as briefly outlined in the following.

Flutter is a self-excited blade vibration whereby an initial small vibration amplitude is amplified through a positive feedback mechanism (negative aerodynamic damping). The flow unsteadiness is only due to blade vibration. This differentiates it from buffeting or NSV, where an aerodynamic unsteadiness exists independent of blade vibration. Classical flutter is an inherently linear phenomenon, where the aerodynamic response scales linearly with vibration amplitude. Non-linearities can arise, for example due to non-linear aerodynamic effects at high vibration amplitudes or friction damping, and result in limitcycle oscillations. In bladed disks, the vibration occurs in one or more nodal diameter patterns resulting in a discrete set of frequencies in the unsteady pressure spectrum. Its signature therefore also differentiates it from NSV.

Buffeting, on the other hand, is caused by an aerodynamic instability. Kielb et al. [11] refer to this as "separated flow vibrations' (SFV) and compare it to the buffeting of wings. In a manner similar to NSV, the aerodynamic frequency locks in with the vibration mode. However, it does not necessarily require a circumferentially travelling disturbance. As a result, it can therefore occur in a 0 nodal diameter (ND) pattern or change in severity over the circumference [12]. If driven by Strouhal instabilities, the buffeting frequency can be estimated from the vortex shedding frequency.

Rotating stall in the sense of Emmon's [13] is a purely aerodynamic phenomenon where stalled operation of one passage gradually triggers adjacent passages. This leads to the formation of full or part-span stall cells which move from passage to passage. The velocity of the stall cell varies between $20 \%$ and $70 \%$ of rotor speed [14], with lower speeds being typical for full span and higher speeds for part-span stall [15]. The speed changes as the cell pattern changes, and the velocity of stall cells is therefore unrelated to the convective time scales of the flow. Its range, however, is comparable to that of the aerodynamic disturbances typically measured during NSV.

Unlike NSV, rotating stall is associated with a significant shift in operating point, resulting from a flow separation on the blade suction side. For the highly three-dimensional flow structure present in modern turbomachinery compressors, this blade boundary layer separation is characterized by locally reversed flow and accordingly a convergence of streaklines. This is not the case in the early stage of NSV. In experiments, radial vortices propagating from blade to blade were observed without flow reversal and without a drop of the compressor performance [16]. The measurements only showed reversed flow conditions after the emergence of a rotating stall cell. This was accompanied by a rapid decrease of blade lift and hence rotor pressure ratio. A clear distinction therefore has to be made between a rotating stall cell and the propagating vortical structures observed during NSV.

Finally, although rotating stall is known to cause blade vibrations [9], there are many examples where a coherent aerodynamic disturbance, i.e. one or multiple stall cells, exists independent of blade vibration. This further differentiates it from the aerodynamic disturbance seen during NSV, which takes a coherent form only after the onset of blade vibrations.

Although not linked to rotating stall itself, NSV has been associated to short length-scale stall precursors, such as 'spikes' and the controversially termed 'rotating instabilities' [17]. These precursors have been an active area of research and are 
known to originate from a variety of aerodynamic features, such as tip clearance vortex oscillations $[18,19]$, leading edge spillage and trailing edge backflow [20,3], leading edge separations [21], shear layer instabilities [22] as well as vortex shedding instabilities [23]. An interaction with blade vibration has been reported in several cases [4, 3, 7] and recently, Brandstetter et al. [16] provided experimental evidence for the lock-in between propagating radial vortices, which are known to be a source of spike-type stall inception through the work of Inoue et al. [24] and Young and Pullan [25, 21], and blisk vibration patterns [16].

Despite the breadth of research on the topic, the physical mechanisms causing NSV and the lock-in of aerodynamic disturbance and structural vibration pattern are not well understood. As a consequence, predicting NSV is extremely difficult. Full-domain computational fluid dynamics (CFD) simulations are possible but unreliable and expensive, precluding use at the design stage.

The main objectives of the current paper are to:

1. Increase the conceptual understanding of the lock-in mechanism leading to NSV

2. Build a reduced model to predict NSV at an early design stage.

These objectives are achieved using numerical simulations of a high speed compressor stage, for which extensive experimental measurements are available. The paper is structured as follows: First, a review of the experimental findings motivating this work is given. The numerical approach is then validated by comparison against the experimental measurements. In the main body of the paper, a parametric study is carried out to identify the factors driving NSV and identify the physical mechanisms responsible. The insights gained from the numerical parameter study are then used to build a simple model, which reflects the physical mechanism. After calibration and validation, it will be shown how the model can be used to predict critical, i.e. unstable, vibration modes.

\section{Review of experimental observations}

In a recent paper, Brandstetter et al. [16] investigated the onset of NSV in a transonic compressor approaching stall using transient wall-pressure, blade strain-gauge and phase-locked PIV measurements at transonic and subsonic conditions. They showed that tip leakage flow creates a large region of low velocity fluid at a (stable) condition near stall, which blocks the passage. In these conditions, small disturbances may lead to the development of unsteady vorticity along the shear layer between the tip leakage and the main flow as shown in Fig. 1 a). These structures have previously been described as radial vortices or tornado vortices because their axis is almost aligned with the radial direction. The vorticity is convected toward the leading edge of the following blade. The trajectory as measured in the experiment is shown in Fig. 1b.

The measurements showed that this aerodynamic disturbance, i.e. the radial vortices, existed in random distribution but with constant propagation velocity long before NSV arose. The

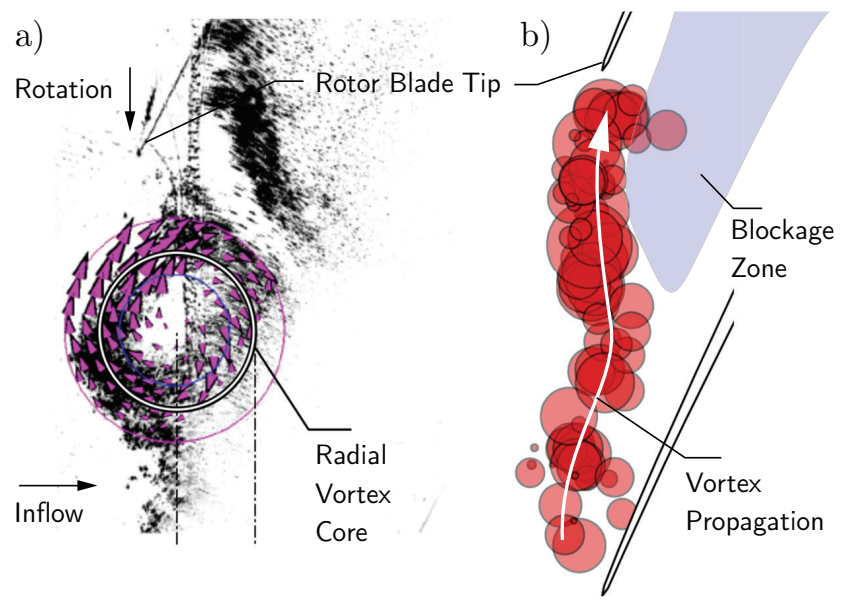

Figure 1: experimental results of transient PIV measurements, a) raw image and relative velocity vectors of a radial vortex during NSV; b) location and size of different radial vortices observed during one transient throttling maneuver; from [16]

propagation velocity differed between experiments at a transonic and a subsonic speedline. During transient throttling manoeuvres, the number and size of the RVs increased. Following the excitation of multiple blade eigenmodes through shedding of radial vortices, a blisk vibration pattern in the first torsional eigenmode developed as the vortex shedding became locked to large blade deflections and a coherent disturbance established. The speed of this disturbance was slightly shifted from the velocity observed before strong vibrations occurred. These measurements suggested that two key ingredients are necessary for NSV:

1. The mean aerodynamics must promote the shedding of radial vortices and their circumferential transport, i.e. the blade must react to small changes in incidence by shedding vorticity while the passage must be blocked to allow the circumferential transport.

2. The blade vibration must be able to modulate the propagation velocity of the aerodynamic disturbance to create a coherent disturbance in resonance with the vibration pattern.

The current paper will use numerical simulations to test these assumptions and increase the conceptual understanding of the lock-in mechanism leading to NSV.

\section{Test case and setup}

\subsection{Transonic compressor}

The test case for this investigation is the Darmstadt transonic compressor, which was experimentally investigated in Reference [16]. It is a modern one-and-a-half stage compressor representative of a high-pressure compressor front stage, designed by Rolls-Royce Deutschland. The rotor has three-dimensional design features like forward-sweep and endwall contouring. Details about the rig operation, inlet guide vanes (IGV) settings, and instrumentation are provided in Ref. [26]. Important 


\begin{tabular}{l|l}
\hline Design pressure ratio & 1.55 \\
Axial inlet Mach number & 0.5 \\
Relative tip Mach number & 1.2 \\
Max shaft speed (rpm) & 20,000 \\
\hline
\end{tabular}

Table 1: Compressor parameters

parameters are summarised in Table 1. This compressor repeatedly suffered NSV in the first torsional mode (1T) near the stall boundary at both transonic and subsonic speed. The 1T mode shape and the corresponding twist deflection of the blade section at $92 \%$ span are shown in Fig. 2. The reduced frequency based on relative inlet velocity, angular frequency and semichord is $k=\frac{\omega_{v} c}{2 v_{\infty}}=1.1$, which is outside the range of typical values for flutter $(k<0.3)$. a)

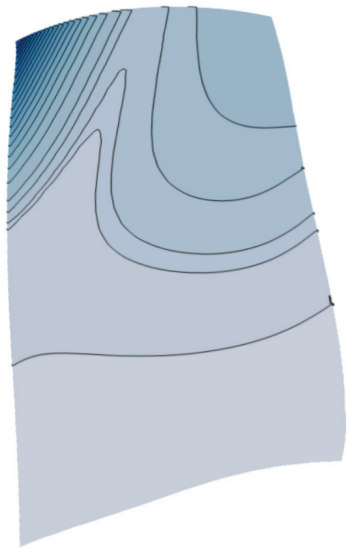

b)

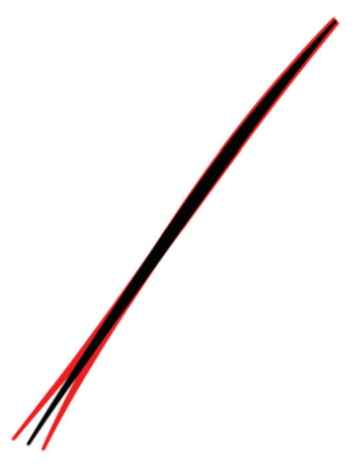

Figure 2: Displacement in first torsional mode shape $(1 \mathrm{~T})$; a) isocontours of modal deformation; b) deformation of blade section at $92 \%$ span

\subsection{Flow solver and model setup}

The flow solver used is AU3D, an unsteady Reynoldsaveraged Navier-Stokes solver for aeroelastic analysis developed at Imperial College with the support of Rolls-Royce. The Spalart-Allmaras turbulence model is used for the present study. The solver has been extensively validated for compressor flows near stall [27, 9] and has also been validated for the current test case [6] using 1.5 stage full circumference simulations. The grid used in this study is comparable to that used by Moeller et al.[6]. It is structured in the radial direction, unstructured in the tangential plane and has approximately 1 million points per rotor passage.

Two sets of steady state simulations are run in preparation for the unsteady analysis. The first one uses the full 1.5 stage domain (see Fig.3) with choked exit nozzle. At the IGV inlet, the measured radial distribution of stagnation pressure, stagnation temperature and flow angles are prescribed as boundary conditions. The second set only contains the rotor and is used as a starting point for the unsteady computations. In this case, the rotor inlet and exit boundary conditions are obtained from the 1.5 stage steady state mixing plane computations. The domain

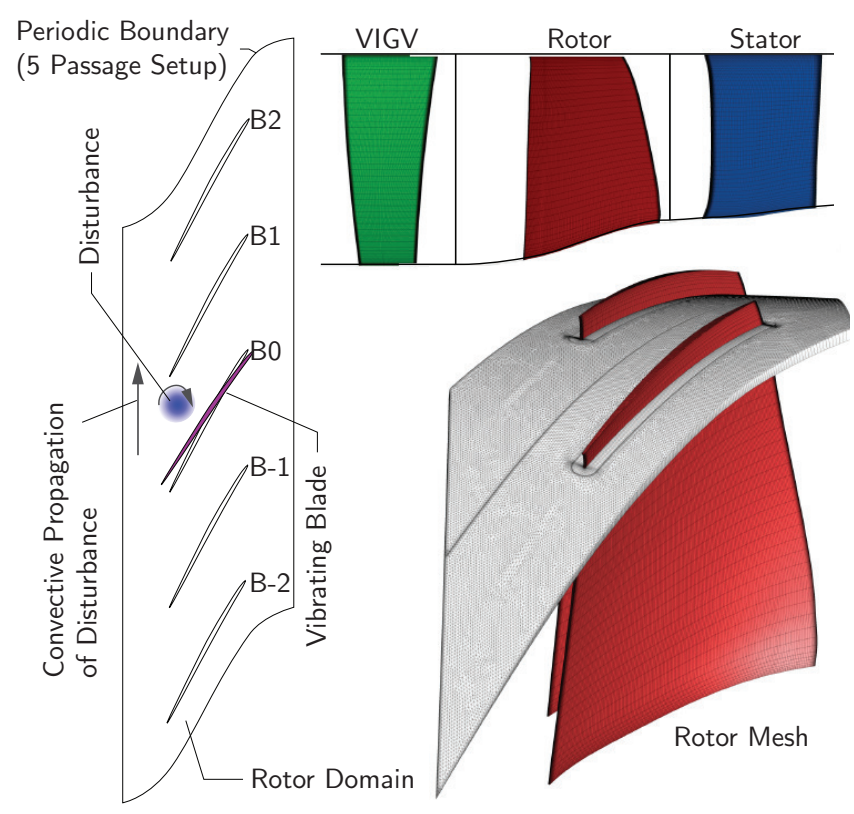

Figure 3: Computational domain for steady and unsteady simulations and detail of rotor mesh

reduction is necessary to facilitate a parameter variation in the unsteady analysis.

For the aeroelastic analysis, the vibration mode shapes are first computed by an in-house finite element solver. In the mode of interest (1T) there is negligible variation with nodal diameter. The mechanical model is therefore reduced to a single mode and weakly coupled aeroelastic analyses are performed, where one blade (Blade 0 ) is undergoing harmonic motion in the $1 \mathrm{~T}$ mode and modal forces on neighbouring blades are recorded. The numerical approach resembles that used to compute aerodynamic influence coefficients (AIC). This will be elaborated on later. Initially, full circumference simulations are carried out. To reduce computational efforts, the domain is then circumferentially truncated to 5 rotor passages with periodic boundary conditions as illustrated in Fig. 3. Results from the truncated domain are compared to the full annulus and it will be seen later that this approach is suitable for the purposes of analysing NSV mechanisms.

The simulations are time-accurate with 200 physical time steps per vibration cycle.

\section{Description and validation of numerical approach}

\subsection{Steady results and determination of unsteady operating point}

The measured and computational constant-speed characteristics, showing total pressure rise across the complete stage, are compared in Fig. 4. The pressure rise across the complete stage matches that of the experiment to within $1 \%$. Figure 4 also shows the pressure rise across the rotor as predicted in the 1.5 stage simulation and in the rotor-only simulation which was throttled further by incrementally increasing the back-pressure. 


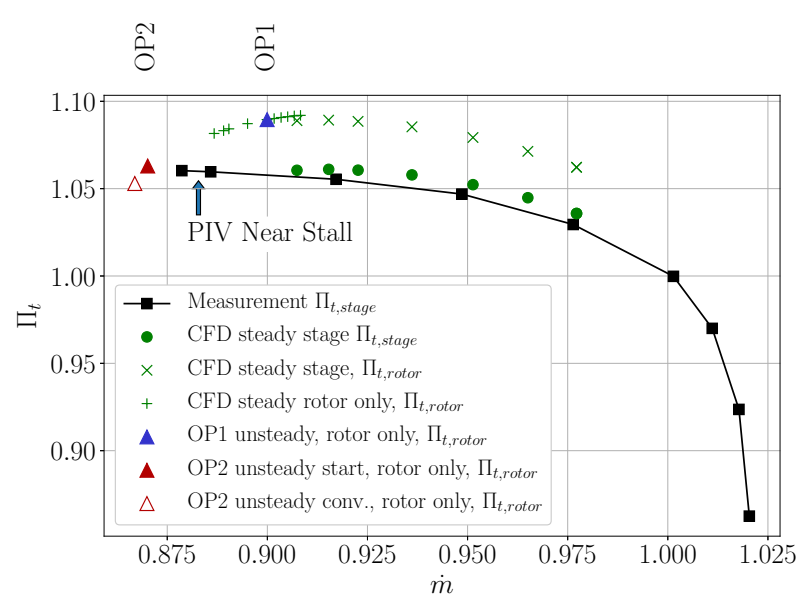

Figure 4: Measured and calculated compressor characteristics; unsteady calculations rotor only; for OP2 (steady) start condition, values normalized with aerodynamic design point

The rotor-only simulation achieves a stall mass flow similar to the experiment.

Two operating points, labelled OP1 and OP2, were selected for the unsteady analysis. OP1 is at the point of maximum total pressure ratio. OP2 is the last point for which a steady state solution could be obtained. The time-averaged operating point shifts slightly in an unsteady simulation, as seen in Fig. 4 but does not yet show the pressure drop associated with rotating stall.

Figure 5 compares the flow field at $92 \%$ span for the two operating points to the experimentally measured flow field near stall, where the first traces of propagating disturbances and NSV were observed. The corresponding operating point is indicated in Fig. 4. The tip flow field of the simulations at OP2 closely resembles the experimental results. The regions of low axial Mach number (blockage regions) created by the tip leakage flow have a similar extent and magnitude. At OP1, on the other hand, the region is noticeably less pronounced and allows a higher velocity near the pressure side close to the leading edge. OP2 was therefore selected as the baseline operating point for the NSV condition. For a full validation of the unsteady simulations, the reader is referred to Reference [6].

Note that, unless otherwise stated, the measurement plane at $92 \%$ span is used throughout the following analysis as this corresponds to the PIV measurement plane [16].

\subsection{Description of aerodynamic disturbance}

In the next step, an unsteady simulation was performed at OP2 in order to recreate the experimental conditions and characterise the aerodynamic disturbance during NSV. During the rig test, aerodynamic disturbances were detected prior to the onset of blade vibration. Reproducing such an initial disturbance numerically requires full circumference simulation over tens of revolutions to allow a build-up of asymmetries. This approach is clearly not suitable for a parametric study. Thank-
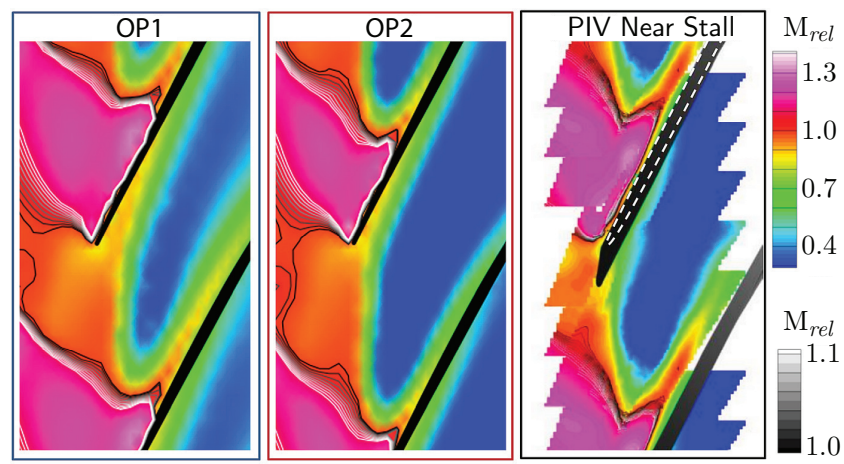

Figure 5: Comparison of relative Mach number at OP1 and OP2 at 92\% Span (pseudo-colours for whole Mach-Number range, grey isocontours in low supersonic range indicating shock structure)

fully, the original trigger is inconsequential for the investigation of the lock-in mechanism. We therefore chose to create the aerodynamic disturbance by harmonically oscillating Blade 0 in the $1 \mathrm{~T}$ mode. The amplitude of vibration was set to $1^{\circ}$ at the blade tip, which was the average value during NSV in the experiments.

The oscillation of Blade 0 creates a periodic aerodynamic disturbance. Figure 6 shows the unsteady velocity field at $92 \%$ span during the first vibration cycle (cycle duration $t_{v}$ ). At this blade height, the vibration mode can be considered as a simple twist of the aerofoil section around mid-chord (see Fig. 2). As the blade accelerates during the oscillation cycle, vorticity is being generated at the solid surface and diffused into the flow, changing the circulation around the blade. The newly generated vorticity is subsequently convected away from the blade. The aerodynamic disturbance can therefore also be described as a flux of vorticity from the blade surface into the flow [28]. Figure 6 shows how vorticity, which is counter-clockwise, is generated during the first part of the vibration cycle. This vorticity travels in the circumferential direction towards the trailing blade as seen in Fig. 6 . The sign of the vorticity will alternate during the vibration cycle as the lift of the blade increases and decreases.

The local drop in static pressure associated with the disturbance is approximately $100 \%$ of the inlet dynamic head. Figure 7a-b show a snapshot of unsteady velocity and unsteady pressure, measured as the difference between the instantaneous static value and the mean of the previous five oscillation cycles. Location and size of these pressured drops compare well to the experimental measurements shown in Fig. 7 c), confirming the aerodynamic similarity of the disturbances and further validating the unsteady simulations.

In order to emphasize the difference between operating condition OP2 and a rotating stall condition which was reached through further throttling, Fig. 8 presents a comparison based on axial velocity contours. At OP2 the surface streaklines do not converge on the suction surface and reversed flow conditions are only observed very close to the casing in the upstream part due to tip leakage flow. The flow field remains symmetric in the downstream part of the blade. For the stalled case, indi- 


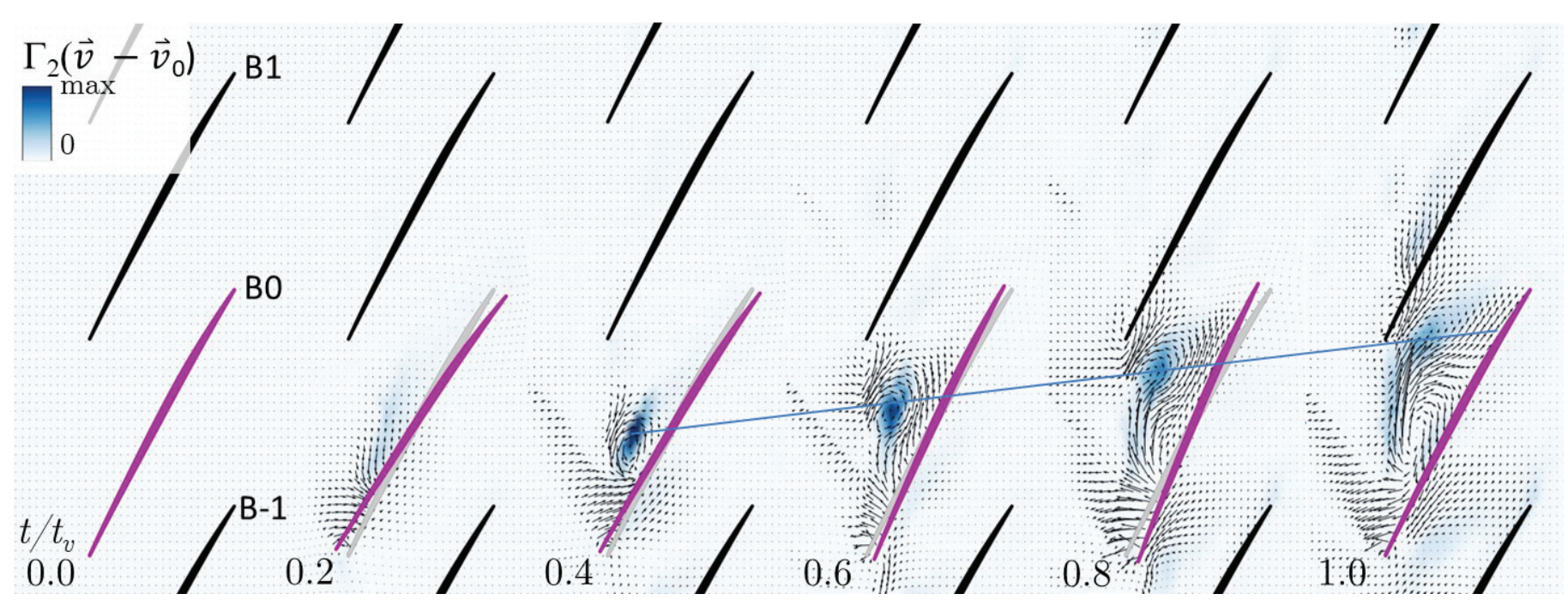

Figure 6: Development of propagating disturbance during one blade oscillation cycle; unsteady velocity vectors and contour map of vortex criterion $\Gamma_{2}$ after Graftieaux et al. [29]

vidual cells are observed which cover more than one blade passage and travel around the circumference. During the passage of a stall cell, the affected blade shows convergent streamlines near the casing with significant backflow in the downstream part.
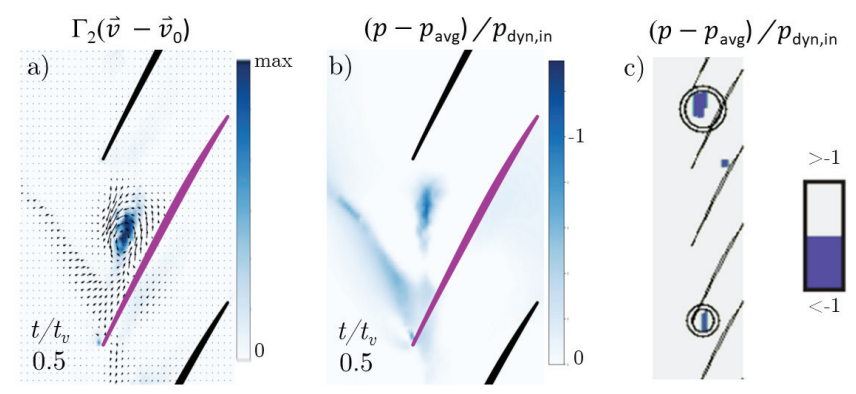

Figure 7: a) unsteady velocity vectors, contourmap of vortex criterion b) contourmap of unsteady pressure, normalized with dynamic head; c) experimental results of measured unsteady wall pressure during NSV from [16]

\subsection{Domain reduction}

To reduce the computational effort, the unsteady simulations are repeated on a sector with only five rotor blades and periodic boundary conditions are applied.

Although the sector size determines the effective number of disturbances in the full $360^{\circ}$ domain, the physical parameters such as propagation speed and amplitude of the disturbance are not affected by the domain truncation. This is confirmed in Fig. 9 which compares the circulation in two passages and snapshots of the unsteady pressure between the five-passage and full annulus domains. The propagation speed was also unaffected by the domain truncation, which can also be deduced from Fig. 9 where the time histories of the two models are in phase.

\section{Analysis at NSV condition: Quantification of distur- bance and link to forcing}

The aerodynamic disturbance in the measurement plane can be quantified by integrating the vorticity, i.e. computing the circulation, over a fixed two-dimensional control volume located mid-passage.

The rationale for this approach is that the vorticity generated at the moving surface is subsequently convected across the control volume contour. While the total amount of circulation will obviously depend on the circumferential extent of the control volume, the change in circulation is therefore a measure of the aerodynamic disturbance generated by the blade oscillation. Examining circulation is useful for relating the disturbance to the aerodynamic forcing of the blades.

As explained before, the vibration mode shape can be viewed as a twist motion around approximately mid-chord for the blade section at $92 \%$ span. The modal force at this section is therefore directly proportional to the section's lift (assuming that the changes are small enough to neglect changes in the location of the centre of pressure). Since both twisting motion and vorticity fluctuations in the flow induce changes in lift, the total forcing can be considered a superposition of the twist-induced forcing, which is zero for non-oscillating blades, and the forcing generated by the vorticity disturbance which is propagating circumferentially.

To test this assumption, Fig. 10 compares the time histories of forcing in the torsional mode $f_{i}$ at OP2 with the circulation measured in the mid-passage control volumes $\Gamma_{i-1, i}$. All values are normalised by a reference amplitude $\hat{\Gamma}_{r e f}$, taken as the amplitude generated by the oscillating blade during its second vibration cycle. It takes approximately five vibration cycles, or 12 blade passing periods $\left(t=12 t_{b p}\right)$, for the disturbance originating from Blade 0 to traverse the domain and return to Blade 0 . A separate variation of the oscillation frequency, which is not described here, has shown that the propagation speed depends 


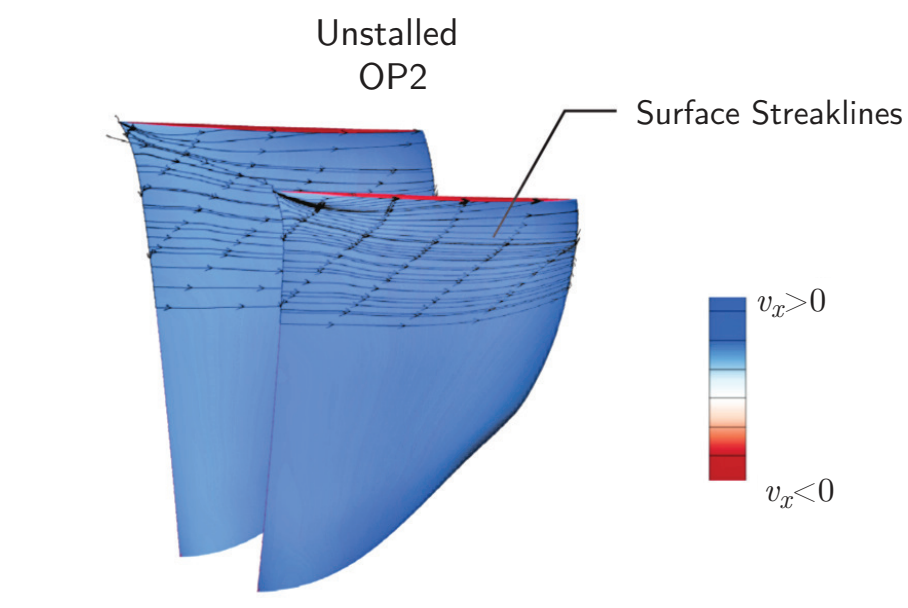

Stalled

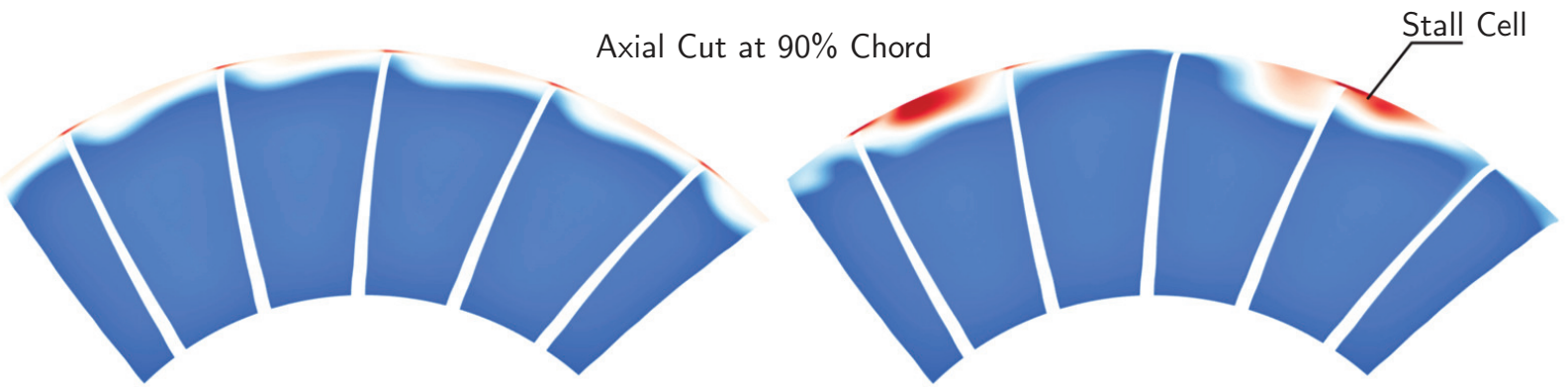

Figure 8: Comparison of flow condition at OP2 and a stalled condition, surface streaklines on blades and axial velocity contour at $90 \%$ chord

only on the rotor aerodynamics and not on the frequency.

For the entire time history, there is a very clear correlation between convected circulation and forcing for all non-oscillating blades. The circulation has been normalised to the initial value measured between Blade 0 and Blade 1, herein after referred to as 'Passage 0,1', but the match in shape between the two curves is remarkable, given that the plots compares circulation at a fixed section of span against total modal force along the complete span. For Blade 0, the dominant forcing in the first 12 blade passing periods results from its own twisting motion. After the disturbance reaches the oscillating blade, the modal force increases in the same manner as for the non-oscillating blades.

Differences between modal force and measured free circulation due to the acoustic/pressure waves are visible in the time histories of Blade 1 and Blade -1 before the convected disturbance reaches the blades. This shows that the acoustic contribution is an order of magnitude smaller than the vorticity component.

The results of this section suggest that: Firstly, the forcing at $92 \%$ span is representative of the three-dimensional case. Secondly, the forcing on non-oscillating blades is predominantly caused by, and can be quantified through, the vorticity disturbance. The acoustic disturbances inevitably created by the blade vibration contribute to the forcing but do not appear to be driving NSV. 
a)

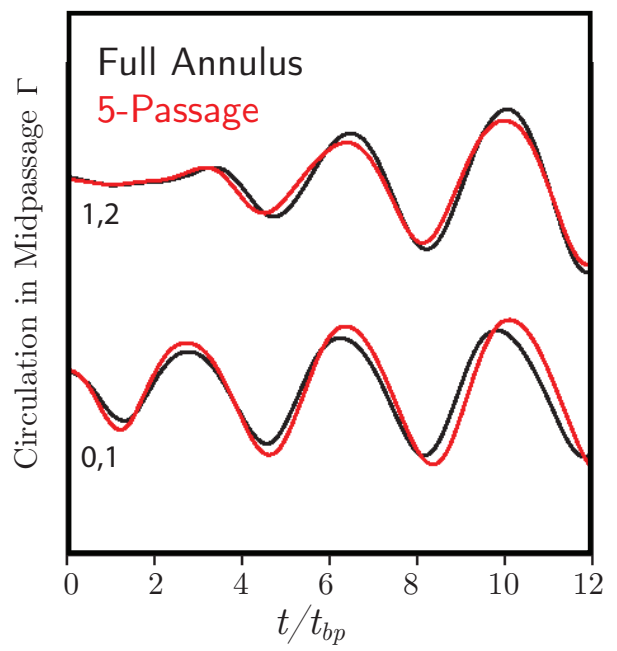

b) Full Annulus

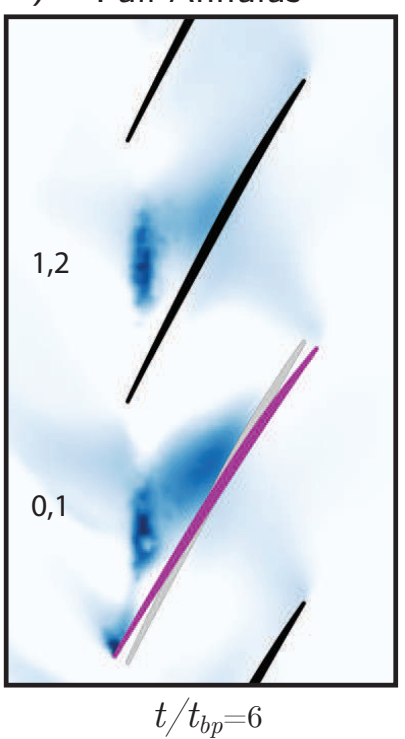

c) 5-Passage

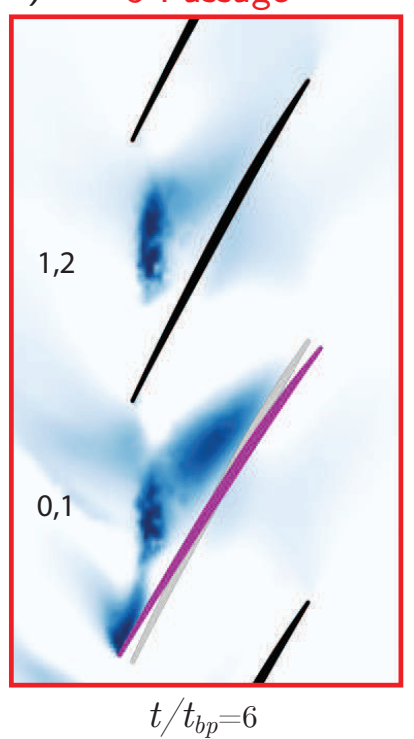

Figure 9: Comparison of five-passage and full annulus domains: a) circulation measured in mid-passage; b) snapshot of unsteady pressure in full annulus setup; c) snapshot of unsteady pressure in five-passage setup

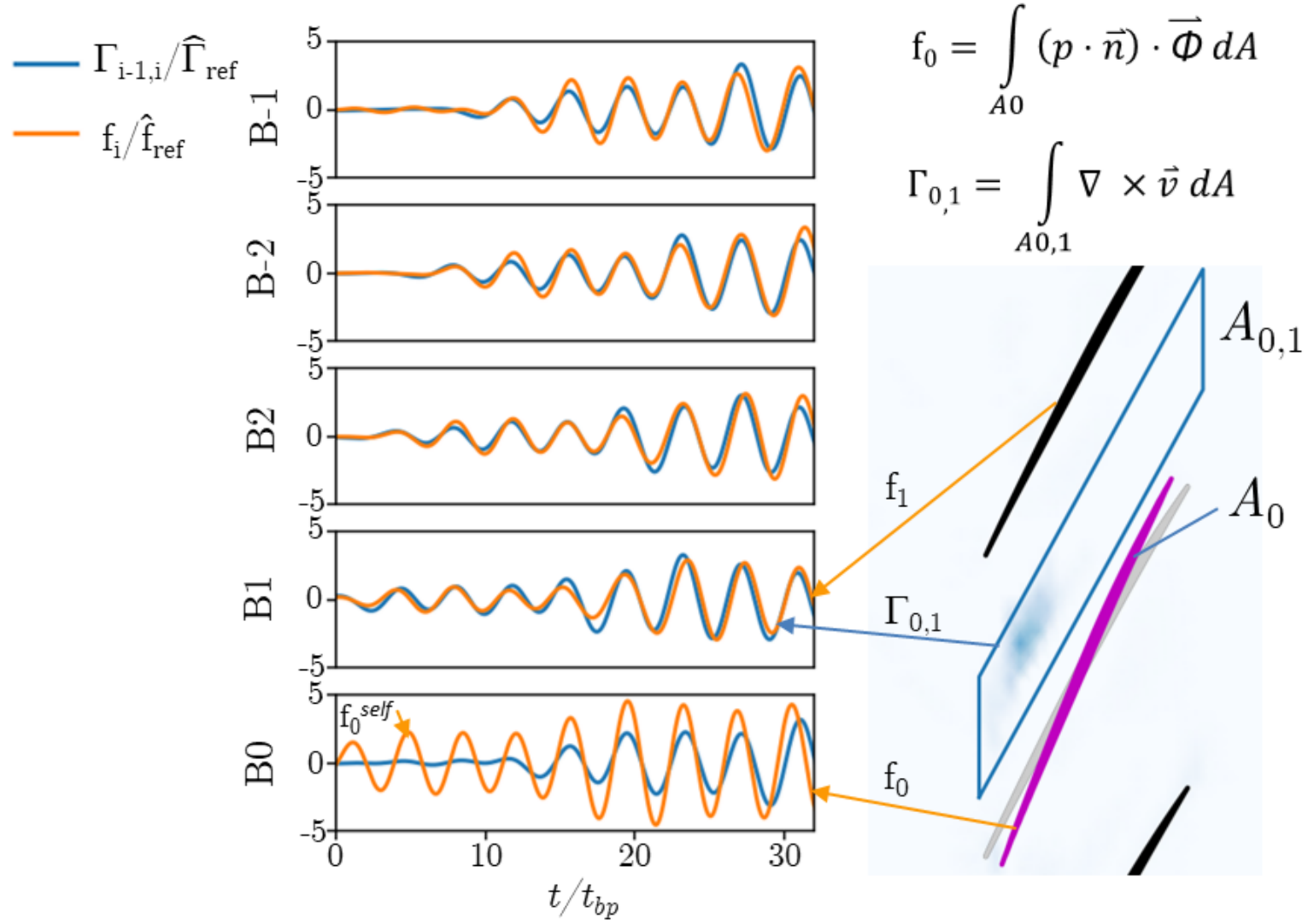

Figure 10: Correlation between convected free circulation and modal forcing at OP2 


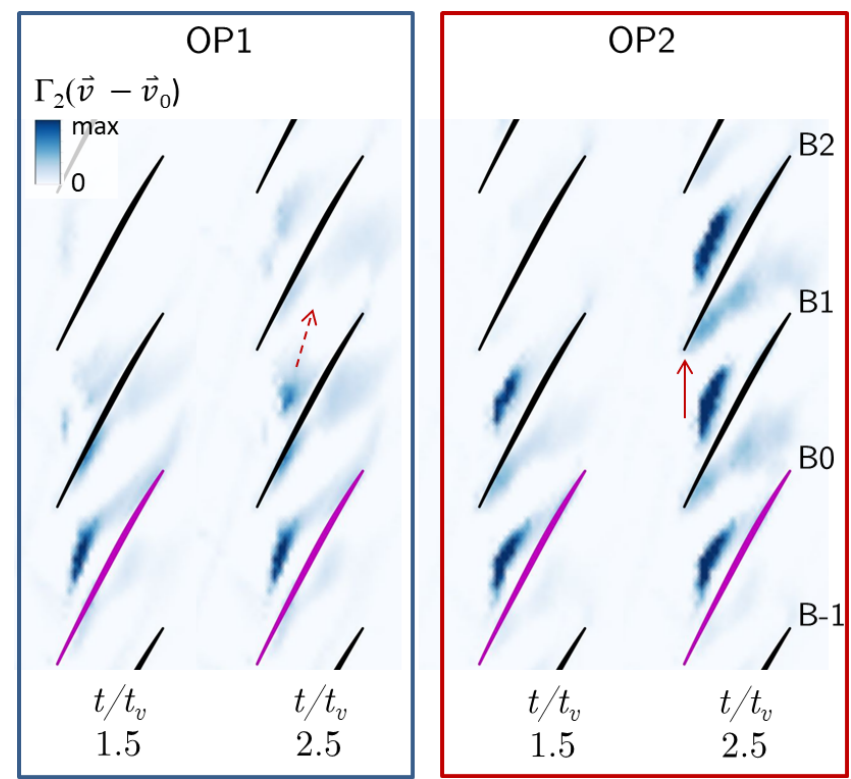

Figure 11: Convective propagation of disturbance, comparison OP1 and OP2, vibrating blade $\mathrm{B} 0$

\section{Parametric study}

To further test the conclusions drawn from the analysis at the NSV condition, the following study investigates the path and strength of the disturbance, the resulting modal forces and their dependency on three parameters: compressor operating point, amplitude of blade vibration and phase between the aerodynamic disturbance and the blade vibration.

\subsection{Effect of Operating Point}

To test the influence of the operating point on disturbance generation, propagation and blade forcing, the unsteady simulation is carried out at the two operating points labelled in Fig. 4 as OP1 and OP2. As previously shown, OP2 is close to the experimental measurement point while OP1 has a slightly higher mass flow rate and smaller tip blockage (Fig. 5). Vibration amplitude and frequency are kept constant.

Figure 11 compares the disturbance between the two operating points by showing snapshots of the vortex criterion $\Gamma_{2}$ proposed by Graftieaux et al [29] inside the domain at two time steps. The disturbance generated in the region between Blade 0 and Blade 1 is slightly stronger at OP2 than at OP1. Furthermore, it travels predominantly into the circumferential direction towards the trailing blade at OP2. At OP1, on the other hand, the vorticity is convected partly axially. The disturbance appearing in Passage 1,2, following interaction with Blade 1, is therefore significantly weaker than that in Passage 0,1. At OP2, the disturbance strength remains approximately constant in the circumferential direction.

As was shown in Section 5, the convected disturbance can be quantified by integrating vorticity over a fixed mid-passage control volume. Figure 12 shows the resulting circulation in each passage over time, and their amplitudes are plotted in Figure 13.
Comparing the results for OP1 and OP2 the observations made above become even clearer. The disturbance measured in Passage 0,1 has a similar amplitude at both operating points, which indicates that there is no significant difference in aerodynamic response to the blade oscillation itself. In the other passages, however, differences become very visible. For OP2, the amplitude does not vary much from blade to blade and at $t / t_{b p} \approx 12$ the initial disturbance arrives in Passage 1,0. For OP1, however, the amplitude decays rapidly and only a weak fluctuation is observed after one domain traversal.

\subsection{Effect of blade vibration amplitude}

To test the relationship between blade vibration amplitude and the resulting disturbance, the amplitude is varied between $0.5^{\circ}$ and $3^{\circ}$ at both operating points. The analysis concentrates on the disturbance during the time period of one domain traversal of the disturbance, i.e. the time between the generation of the first vorticity fluctuation and its arrival at the oscillating blade, in order to avoid ambiguity caused by the superposition of incoming and newly generated vorticity fluctuations.

Figure 14 compares the magnitude of the aerodynamic disturbance in Passage 0,1 and Passage 1,2 for different vibration amplitudes, $\hat{\alpha}$. At OP1, the relationship between disturbance and amplitude is linear. At OP2 the linear relationship holds until approximately $1^{\circ}$ but for larger amplitudes, the disturbance is saturated because the blade is operating close to stall. At this condition, there is a drop in vorticity diffused from the moving blade surface. Similarly, the vorticity generated from the trailing blade in response to an incoming vorticity disturbance is limited. The maximum modal forcing resulting from the convected vorticity is therefore limited by the rotor's aerodynamic characteristics. This non-linear behaviour at high amplitudes leads to the development of limit-cycle oscillations in the experiment.

The results discussed above apply to the time period before the propagating disturbance interacts with the oscillating blade. From Fig. 12, it is clear that the amplitude of the disturbance remains constant during an interaction with a fixed blade at OP2, while it is gradually reduced at OP1. Obviously, the development of the disturbance upon interaction with an oscillating blade will depend on the phase difference between the vibration and the vorticity disturbance.

\subsection{Effect of interaction phase}

To characterize this dependency the interaction of a vorticity disturbance with an oscillating blade was studied for different relative phases between the disturbance and the blade vibration. This was achieved with the same setup by changing the vibration frequency. Since the propagation velocity and amplitude remain unchanged $(<0.5 \%)$, this changes the distance travelled by the disturbance in one vibration cycle and therefore the vibration phase at the time of interaction. This phase, also referred to as interaction phase in the following, is given by:

$$
\frac{\varphi}{2 \pi}=\frac{\omega_{v}}{2 \pi} t_{\text {trav }}=\frac{N_{b, \text { domain }}}{N_{b}} \frac{\omega_{v}}{\Omega_{a}^{R}}
$$




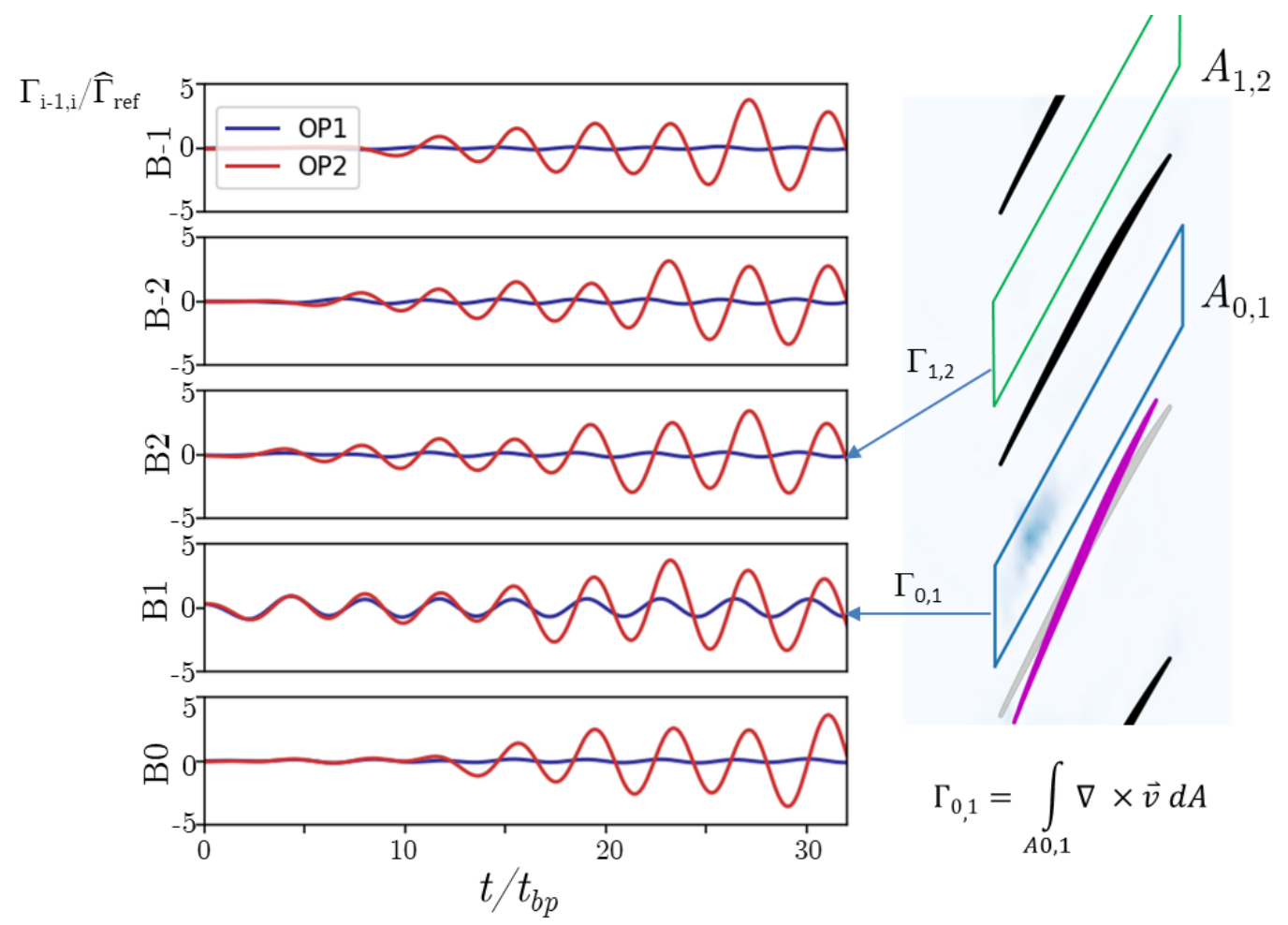

Figure 12: Convected Circulation, Comparison OP1 and OP2 for modal deflection amplitude $\hat{\alpha}=1^{\circ}$ at blade tip

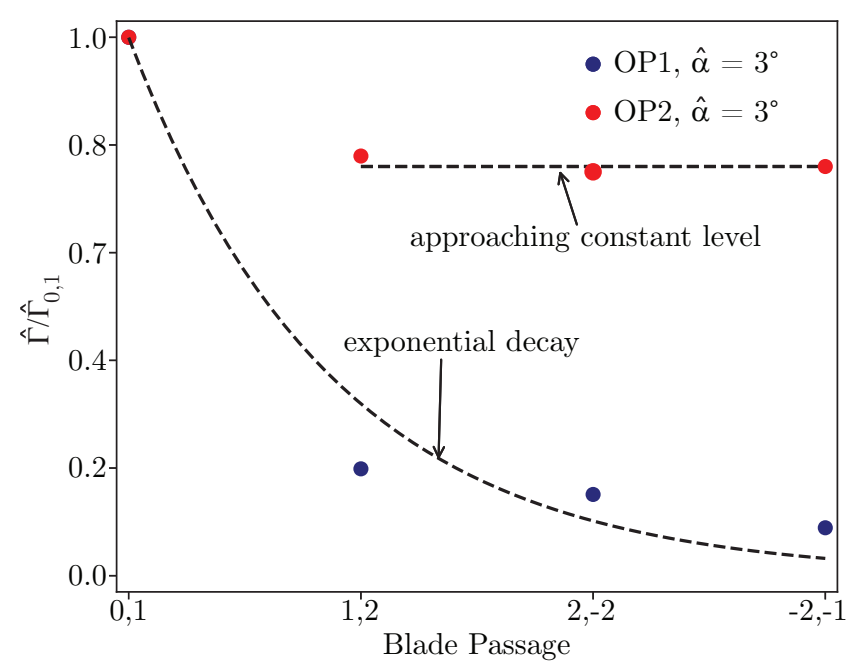

Figure 13: Passage-to-passage variation in amplitude, Comparison OP1 and OP2 for modal deflection amplitude $\hat{\alpha}=3^{\circ}$ at blade tip

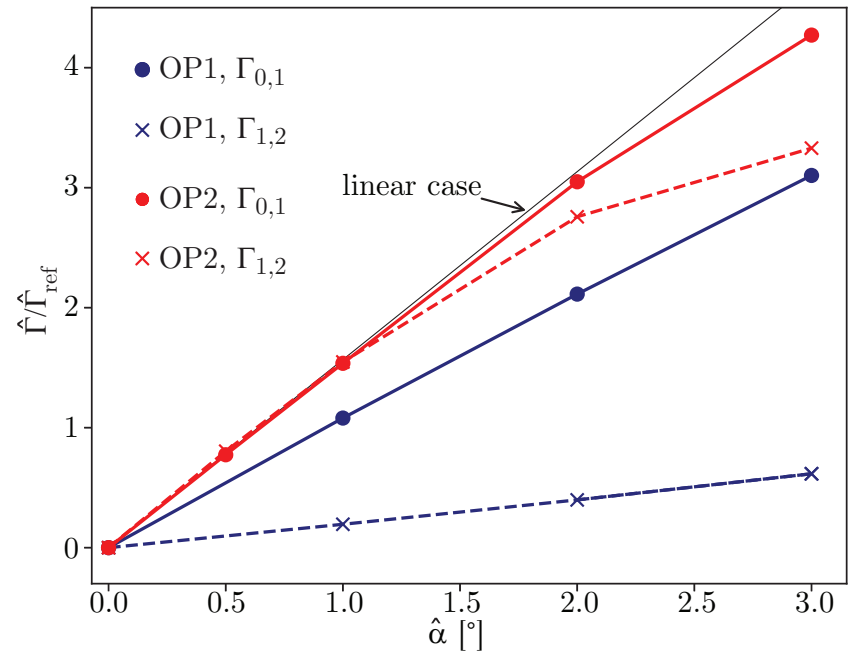

Figure 14: Amplitude of the aerodynamic disturbance for different vibration amplitudes at operating point OP1 and OP2, measured in Passage 0,1 and 1,2; normalized with amplitude of OP1 for $\hat{\alpha}=1^{\circ}$ 
where $t_{\text {trav }}$ is the time taken for one domain traversal of the disturbance, $\omega_{v}$ is the vibration frequency, $N_{b \text {,domain }}$ is the number of blades in the domain and $\Omega_{a}^{R}$ is the angular velocity of the disturbance in the rotor frame of reference.

Figure 15a shows the disturbance amplification after interaction with an oscillating blade for various interaction phases. As to be expected, the trend is sinusoidal. The maximum amplification occurs when the disturbance is leading the blade by approximately $1 / 5$ th of a cycle, i.e. when the blade is opening as positive circulation arrives at the blade location. The minimum amplification occurs when the blade is starting to close.

In addition to amplifying the disturbance, the interaction also modulates the phase of the disturbance. When the incoming vorticity disturbance is out of phase with the blade motion, it results in a delay of vorticity being convected from the surface. This can be explained as follows: In undisturbed inflow, the blade oscillation results in the periodic generation of vorticity at the blade surface and its subsequent convection into the passage. This periodic process is altered if the convected vorticity disturbance reaches the oscillating blade and changes its inflow conditions. In response to the change in conditions, the rate of diffusion of vorticity from the solid surface and consequently the circulation measured mid-passage adjusts.

The interaction therefore effectively alters the local wave length and frequency. It can be thought of as a local phase modulation, or change in phase velocity, of the disturbance by the vibration. Figure $15 \mathrm{~b}$ shows how this modulation varies with interaction phase. Again, the trend is sinusoidal as expected, and it correlates well with the amplitude variation shown in Fig. 15a.

Although the free convective velocity of the flow has not changed, the shift appears to cause a change in the velocity when measured globally by tracking disturbance troughs. In the following sections, these two velocities will be referred to as 'free' and 'global' propagation velocity respectively.

The amplitude range of Fig. 15a furthermore confirms that the disturbance generated by the interaction of an incoming disturbance with an oscillating blade can effectively be considered a superposition of the incoming disturbance, and the disturbance generated by the blade oscillation.

\subsection{Conclusions from the parametric study}

The results presented in this parametric study clearly demonstrate that the forcing causing NSV is generated by a circumferentially convected aerodynamic disturbance, which is quantifiable in terms of circulation within the blade passages. The path of the disturbance is determined by the mean flow conditions and a partially blocked passage is necessary to enable the disturbance to propagate circumferentially.

The forcing on a vibrating blade was found to be a superposition of the force induced by the vibration itself and that caused by the incoming disturbance and was shown to be proportional to the resulting changes in circulation. Whether or not the superposition of the forces leads to an increase or decrease in amplitude depends on the relative phase of the vibration and the incoming disturbance.
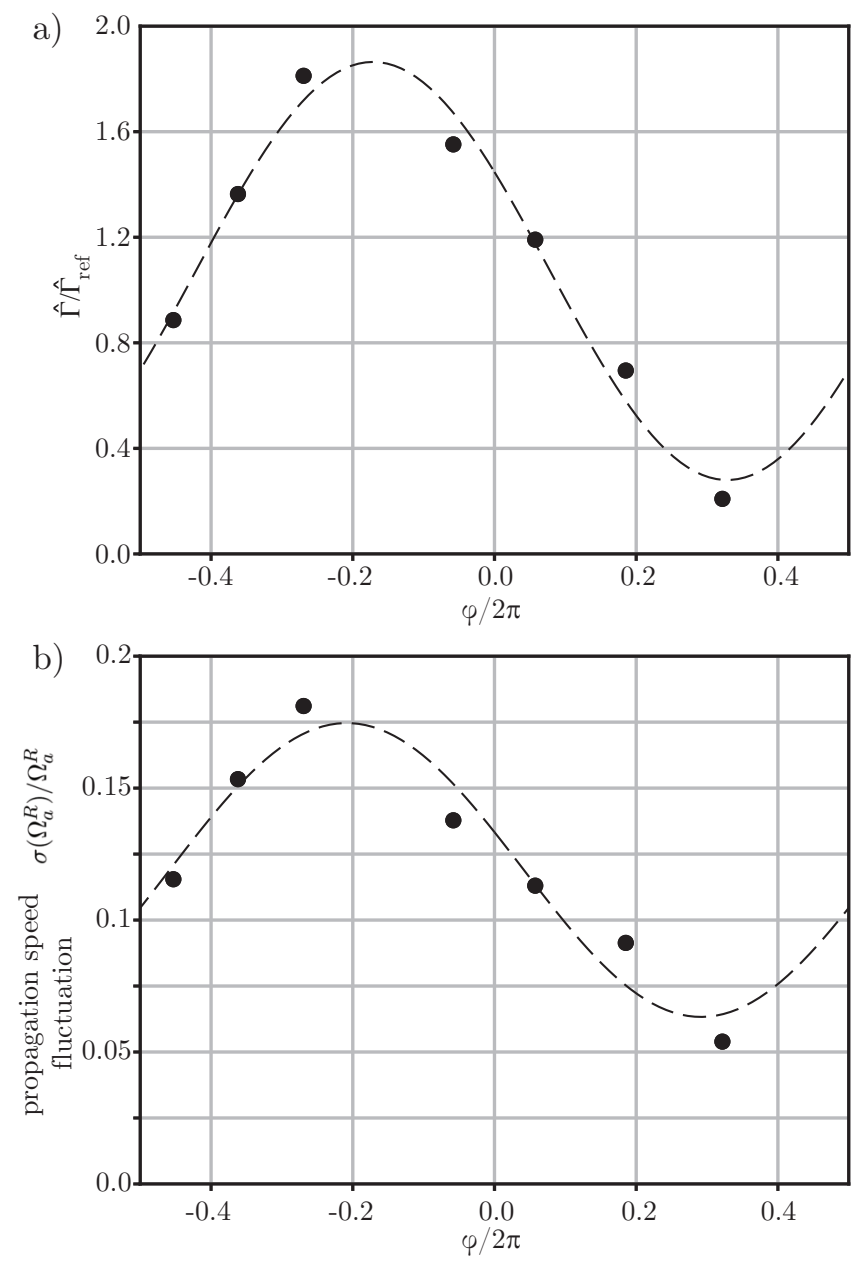

Figure 15: Effect of interaction phase on amplitude and propagation speed 
The interaction of the vorticity disturbance and the oscillating blade was shown to locally alter the phase of the disturbance, which leads to an effective change in propagation velocity. This modulation and amplification of the disturbance creates the lock-in effect seen in the experimental measurements, where onset of NSV was linked to the transition from broadband to coherent disturbances and associated small changes in propagation speed.

\section{Semi-analytical model}

Using the knowledge gained from the parametric study, we developed a semi-analytical model for NSV. Semi-analytical models have in the past proved useful for delivering insights and preliminary design predictions into rotor vibrations, e.g. [30] and [31]. The present model serves three purposes: Firstly, it will test the conclusions drawn from the CFD model regarding the nature of NSV. Secondly, it will allow a fast parameter sweep to further investigate the relationship and lock-in between aerodynamic disturbance and vibration mode. Lastly, it will serve as a tool to predict critical vibration modes at an early stage during design.

\subsection{Model derivation}

The model uses single-degree of freedom mass oscillators to represent blades on a rigid disk, as illustrated in Fig. 16. The equation of motion for the system is given by:

$$
\ddot{\mathbf{q}}(t)+D \dot{\mathbf{q}}(t)+K \mathbf{q}(t)=\mathbf{f}(t)
$$

where $q(t)$ is the modal displacement vector and $f$ is the massnormalized modal force vector.

If the blades are mechanically uncoupled, $D=\operatorname{diag}\left[2 \zeta \omega_{v, i}\right]$ is the diagonal damping matrix and $K=\operatorname{diag}\left[\left(\omega_{v, i}\right)^{2}\right]$ is the diagonal stiffness matrix.

Since the CFD results have shown that a quasi-2D analysis of the aerodynamic disturbance in the measurement plane is appropriate, we will consider a 2D blade section undergoing pure twist motion around the blade torsional axis to find an expression for the forcing term in Eq. 2. This aids understanding but with appropriate tuning the model can be applied to general cases and other mode shapes.

For a pure twist mode, the modal force on the right hand side can be replaced by the moment induced by the unsteady lift and the modal displacement can be replaced with twist of the blade $\alpha \propto q$. If the changes in the location of the centre of pressure are negligible, Eq. 2 becomes:

$$
\ddot{\boldsymbol{\alpha}}(t)+D \dot{\boldsymbol{\alpha}}(t)+K \boldsymbol{\alpha}(t)=c_{1} \boldsymbol{L}^{\prime}
$$

where $c_{1}$ is a constant of proportionality between the $2 \mathrm{D}$ unsteady lift, $L^{\prime}$, and 3D mass-normalised modal force. Note that in this particular case, the modal force is a moment but to align with convention, we shall refer to it as modal force. The results of the previous section are now used to derive an expression for the unsteady lift, $L^{\prime}$.
The unsteady simulations have shown that the integral modal force, and therefore lift, $L_{i}$ on Blade $i$ can be considered a superposition of the force induced directly by the blade oscillation, $L_{i, i}$, and that caused by the incoming vorticity disturbance, $L_{i, \Gamma_{i}}$ :

$$
L_{i}^{\prime}=L_{i, i}^{\prime}+L_{i, \Gamma_{i}}^{\prime}
$$

We begin by deriving an expression for the forcing caused by the blade motion. Following Theodorsen [32] and assuming that apparent mass effects are negligible, that the centre of torsion is located at half-chord and that the twist velocity is negligible compared to the relative inlet velocity, $\dot{\alpha}<<v_{\infty}$, the span-specific unsteady lift of Blade $i$ for a given operating point can be written as:

$$
L_{i, i}^{\prime}(k, t)=\bar{L}_{\theta}(k) \frac{\rho v_{\infty}^{2} c}{2} \alpha_{i}(t)
$$

where $c$ is the blade chord and $k$ the reduced frequency. The complex function $\bar{L}_{\alpha}(k)$ is Theodorsen's function and models the unsteady aerodynamic response of the blade. It will also depend on the operating point. In the following, we will assume a constant operating point and tuned system to drop dependency on $k$. We also drop the subscript $i$ for brevity.

The CFD simulations have shown that the forcing generated by the aerodynamic disturbance is proportional to the circulation convected from blade to blade in the $2 \mathrm{D}$ plane. It is therefore appropriate to use the Kutta-Joukowski theorem to approximate the force induced by a travelling disturbance $\Gamma(\theta, t)$ as:

$$
L_{i, \Gamma_{i}}^{\prime}(t)=\bar{L}_{\Gamma} \rho v_{\infty} \Gamma\left(\theta_{i}, t\right)
$$

where $\theta_{i}$ is the circumferential position of Blade $i$ and, as above, $\bar{L}_{\Gamma}$ is a complex constant modelling the change in amplitude and phase relative to the quasi-steady force induced by the convected circulation.

The total unsteady lift for the 2D section can therefore be written as:

$$
L_{i}^{\prime}(t)=\bar{L}_{\alpha} c \frac{\rho v_{\infty}^{2}}{2} \alpha_{i}(t)+\bar{L}_{\Gamma} \rho v_{\infty} \Gamma\left(\theta_{i}, t\right)
$$

To complete the model, we now need an expression for the circulation $\Gamma(\theta, t)$. This requires a model for the generation and convection of the disturbance around the circumference.

To model the circumferential transport we consider the rate of change of circulation in an arbitrary fixed control volume $A$ in the x-y measurement plane at constant $R$ where $y=R \theta$. By integrating the two-dimensional vorticity transport equation for viscous, incompressible flow over this control volume we obtain:

$$
\frac{d \Gamma_{A}}{d t}=-\int_{A}[(\mathbf{v} \cdot \nabla) \mathbf{w}] d A+\int_{A} v \nabla^{2} \mathbf{w} d A+\dot{\Gamma}_{S}
$$

where $\mathbf{w}=\left[\begin{array}{lll}0 & 0 & \mathbf{w}_{z}\end{array}\right]$ considers only the vorticity in the measurement plane, $v \nabla^{2} \mathbf{w}$ is the viscous diffusion across the control volume contour and $\dot{\Gamma}_{S}$ is a source term used to model the generation of vorticity at the oscillating blades, the exact form of which will be described shortly. The transport is modelled in 


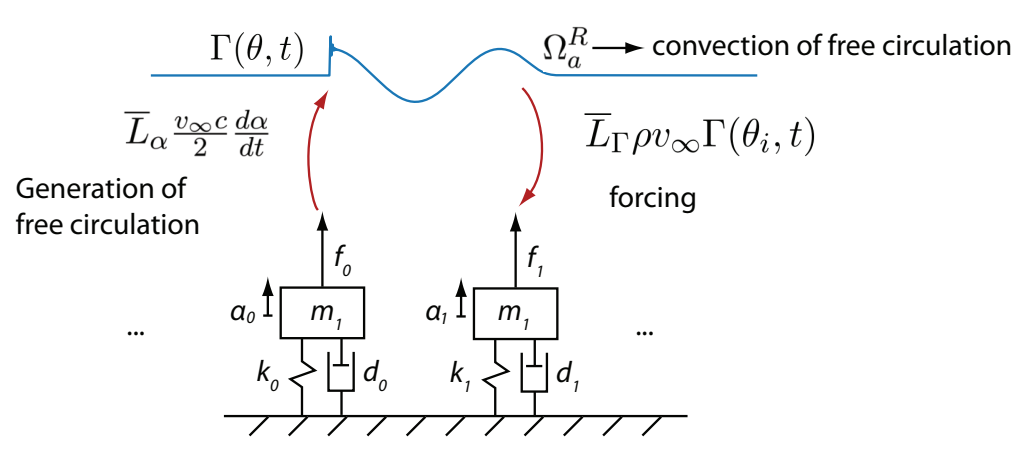

Figure 16: Schematic of Semi-analytical Model

the relative frame, with the relative velocity given by $\mathbf{v}$. The $2 \mathrm{D}$ assumption neglects any radial convection, radial variation and vortex stretching. It is therefore a radically simplified model of the disturbance propagation and not intended to model the true evolution of vorticity in a blade row.

Since only the circumferential transport is of interest to NSV, we choose a rectangular control volume spanning the length of the passage with its sides $L_{x}$ and $L_{y}$ aligned with the axial and circumferential direction respectively. This allows the convection and diffusion across the circumferential sides to be grouped together in a sink term, such that after applying Stokes' theorem Equation 8 simplifies to

$$
\frac{d \Gamma}{d t}=-\int_{L_{x}} \int_{L_{y}} \mathrm{v}_{y} \frac{\partial \mathrm{w}_{z}}{\partial y} d y d x+\int_{L_{x}} \int_{L_{y}} v \frac{\partial^{2} \mathrm{w}_{z}}{\partial y^{2}} d y d x+\dot{\Gamma}_{S}+\dot{\Gamma}_{s i n k}
$$

$\dot{\Gamma}_{\text {sink }}$ is the sink term which accounts for the loss of vorticity axially out of the rotor domain. It depends on the operating point and can be calibrated from the CFD simulations. This term is generally a non-linear function, but for low disturbance amplitudes the linear approximation $\dot{\Gamma}_{\text {sink }}=C_{s} \Gamma$ was found to be appropriate for a preliminary assessment. For modelling the saturation of disturbance seen in the simulation and limit-cycle oscillations, the non-linearity would have to be included.

The source term $\dot{\Gamma}_{S}$ is zero everywhere but at the blade locations $\left(\theta_{i}\right)$, where it models the vorticity generated by the blade movement. It can be approximated by considering that the rate of change of total vorticity flux from the blade is equal to its rate of change of circulation (see Eq. 5):

$$
\dot{\Gamma}_{S}(\theta, \alpha(t))= \begin{cases}\bar{L}_{\alpha} \frac{v_{\infty} c}{2} \frac{d \alpha}{d t} & \text { for } \theta=\theta_{i} \\ 0 & \text { for } \theta \neq \theta_{i}\end{cases}
$$

where $\theta_{i}$ is the location of Blade $i$. Similar to the sink term, the vorticity generation behaves non-linearly at high vibration amplitudes as seen in Fig. 14. This is neglected in the current formulation but will be incorporated in the model in future work.

By assuming a constant angular velocity of the disturbance, $\Omega_{a}^{R}$, Eq. 9 simplifies to the following one-dimensional equation, describing the rate of change of circulation in fixed control vol- umes placed around the circumference:

$$
\frac{d \Gamma}{d t}=-\Omega_{a}^{R} \frac{\partial \Gamma}{\partial \theta}+\frac{v}{R} \frac{\partial^{2} \Gamma}{\partial \theta^{2}}+\dot{\Gamma}_{S}(\theta, \alpha(t))+\dot{\Gamma}_{s i n k}
$$

In a first step, assuming no source of unsteady circulation but the term proportional to the blade oscillation, we define $\Gamma^{\prime}=2 \Gamma /\left(\bar{L}_{\alpha} v_{\infty} c\right)$ to simplify the equation of motion, such that substituting Eq. 7 into 3 results in:

$$
\ddot{\boldsymbol{\alpha}}(t)+D \dot{\boldsymbol{\alpha}}(t)+K \boldsymbol{\alpha}(t)=\frac{1}{2} c_{1} \rho v_{\infty}^{2} c \bar{L}_{\alpha}\left[\boldsymbol{\alpha}(t)+\bar{L}_{\Gamma} \boldsymbol{\Gamma}^{\prime}(t)\right]
$$

which, together with Eq. 11, forms a coupled system. The bidirectional interaction between the disturbance and an oscillating rotor blade arises from the source-term in Eq. (11) and the second forcing term in Eq. (12). The convection of the circulation couples the different rotor blades aerodynamically.

In its current form Eqn. 12 describes an aerodynamically tuned system. To correctly model a system with frequency variations, the complex constants must be replaced by frequencydependent functions.

This system is discretised in one dimension around the circumference and numerically integrated in time using an implicit time-marching scheme.

Before the model is tested, the constants, summarized in Table 2 are calibrated using the CFD simulations.

The magnitude of the proportionality term $c_{1}$ can be deduced from the modal forcing amplitude $\hat{f}_{1,0}$ on the oscillating blade prior to interaction with a convected disturbance (see Fig. 10). The phase of $\bar{L}_{\alpha}$ can be directly derived through comparison of $f_{1,0}(t)$ and $\alpha_{0}(t)$. The propagation velocity of the disturbance $\Omega_{a}^{R}$ is obtained through tracking of the initial disturbance (Fig. 6). The two constants $\bar{L}_{\Gamma}$ and the circulation loss function $\dot{\Gamma}_{\text {sink }}$ are fitted using a least square regression to match the modal forcing on non-oscillating blades observed in the simulations over two domain traversals of the disturbance (Fig. 10 and Fig. 13).

\subsection{Comparison between NSV model and linear flutter models}

In the model shown above, the aerodynamic forcing results only from the blade vibration and is linearly dependent on the blade vibration amplitude. It is possible to transfer it into the frequency domain and predict stability using eigenvalue analysis, as for classical flutter models. Moreover, the numerical 


\begin{tabular}{l|l}
\hline$\Omega_{a}^{R}$ & $\begin{array}{l}\text { propagation speed of disturbance } \\
\text { Transfer from modal oscillation to lift on oscillat- } \\
\bar{L}_{\alpha}\end{array}$ \\
$\bar{L}_{\Gamma}$ & $\begin{array}{l}\text { ing blade } \\
\text { Transfer from convected circulation to modal } \\
\text { force }\end{array}$ \\
$\dot{\Gamma}_{\text {sink }}$ & $\begin{array}{l}\text { Loss of circulation due to axial convection and } \\
\text { diffusion, generally a non-linear function but can } \\
\text { be modeled for low amplitudes as } \dot{\Gamma}_{\text {sink }}=C_{s} \Gamma, \\
\text { for higher amplitudes (tested up to } 3^{\circ} \text { ) modeling } \\
\text { as } \dot{\Gamma}_{\text {sink }}=C_{s 1} \Gamma+C_{s 2} \Gamma^{2} \text { allows the consideration } \\
\text { of a saturation of circulation and leads to a limit } \\
\text { cycle behavior of the system. }\end{array}$ \\
\hline
\end{tabular}

Table 2: Parameters and their purpose in the semi-analytical model

approach used for calibration is also similar to aerodynamic influence coefficient (AIC) methods and in the case of OP1, where the disturbance decays over the circumference, it is possible to use the AIC method for stability prediction. However, in the case of OP2, where the aerodynamic disturbance does not decay significantly over the circumference and therefore returns to Blade 0 , the fundamental assumption at the origin of the AIC method, namely that far away blades have no influence on the vibrating blade itself, is invalidated. A frequencyrepresentation of the model to further illustrate the differences between a NSV and flutter model is out of scope of this paper but the time-marching method formulated here has the advantage that it allows a transient analysis of the system behaviour. With some modifications, it is also possible to include nonlinearities in the sink and source term and to manipulate the disturbance in time and space. The latter can be used to model geometric asymmetries in the rotor or additional modulations of the forcing term, for example due to intake distortions or casing treatments.

\section{Result from semi-analytical model}

\subsection{Validation}

In the first step, the model is validated against the reduceddomain CFD simulations. It is set up with five blades, or five lumped mass oscillators, and the first blade is harmonically oscillated, while all other blades remained fixed.

The modal stiffness is set to that of the $1 \mathrm{~T}$ mode, which normalized by the shaft frequency of this rotor is $\omega_{v}=5.82 \Omega_{r}^{R}$. Damping was set to $\zeta=0.0001$. The model is then calibrated for operating point OP2 with an interaction phase of $\varphi=-\pi / 2$ and a blade vibration amplitude of $\hat{\alpha}=1^{\circ}$.

Figure 17 compares the resulting blade forcing to the CFD simulations. As expected, they match well for the calibrated vibration frequency and the associated interaction phase (Fig. 17a). When the oscillation frequency and hence interaction phase are varied but parameters remain the same, the model still adequately reproduces the CFD results. This is shown in Fig. 17b for the most extreme change in interaction phase $\varphi=+\pi / 2$, where the disturbance histories match well on all blades, even at the time of impact of the disturbance on the oscillating Blade 0 at $t / t_{b p}=18$. Other phases across the complete range matched similarly well. There is a small difference at Blade -1 before the arrival of the convected disturbance at Blade -1 . This is caused by acoustic forcing which is present in the CFD but neglected in the current model. The fact that the modal force is nevertheless well approximated confirms that the contribution from the convected part is dominant. It therefore further justifies the neglect of acoustic forcing in the model and in the description of the NSV mechanism in the previous section and the almost linear system behavior for low vibration amplitudes.

\subsection{Application to flexible full annulus rotor}

After this validation against the CFD results, the model is applied to the same test case but the blade movement is not prescribed and all 21 blades are included. The unsteadiness is triggered with an initial disturbance at Blade 16 and the resulting blade displacements and disturbance locations are tracked. Figure 18 presents the time history of the solution, with the blade oscillations shown as blue lines and the track of the propagating disturbances in grey. It is clear that the disturbance from Blade 16 propagates in circumferential direction and excites the trailing blades. After approximately two revolutions the initial disturbance has travelled around the circumference.

Every time the disturbance interacts with an oscillating blade, its path is slightly shifted. A zoomed view of this interaction is shown in Fig. 19a. Here the shift of the disturbance at each interaction is clearly visible. This leads to a change of the global propagation speed, which is recorded in Fig. 19b. It clearly shows the acceleration after the first domain traversal. After approximately 6 revolutions, the propagation speed stabilizes to a value approximately $2.5 \%$ higher than the free propagation speed. At this point the system is considered locked in. The frequency and inter-blade phase angle $\left(\sigma_{a}=2 \pi N_{a} / N_{b}\right)$ of the aerodynamic disturbance match those of the structural vibration:

$$
\begin{gathered}
\omega_{v, l o c k}=\omega_{a, l o c k}=N_{a} \Omega_{a, l o c k}^{R} \\
\sigma_{v}=\sigma_{a} \rightarrow N D \equiv N D_{a} \quad\left(\bmod N_{b}\right)
\end{gathered}
$$

In other words, a coherent aerodynamic disturbance and structural nodal diameter pattern are established. The model has shown that this lock-in is achieved by adjustment of the global propagation speed, $\Omega_{a}^{R}$, and selection of a matching nodal diameter pattern and aerodynamic wave number. The vibration mode and shift in propagation speed match those of the experiment.

\subsection{Sensitivity analysis and prediction of critical vibration modes}

The previous model application provided a prediction of the critical vibration mode for a known propagation speed, $\Omega_{a}^{R}$. This can be approximated from steady state computations but it will differ from different rotational speeds and guide vane settings. 
a)

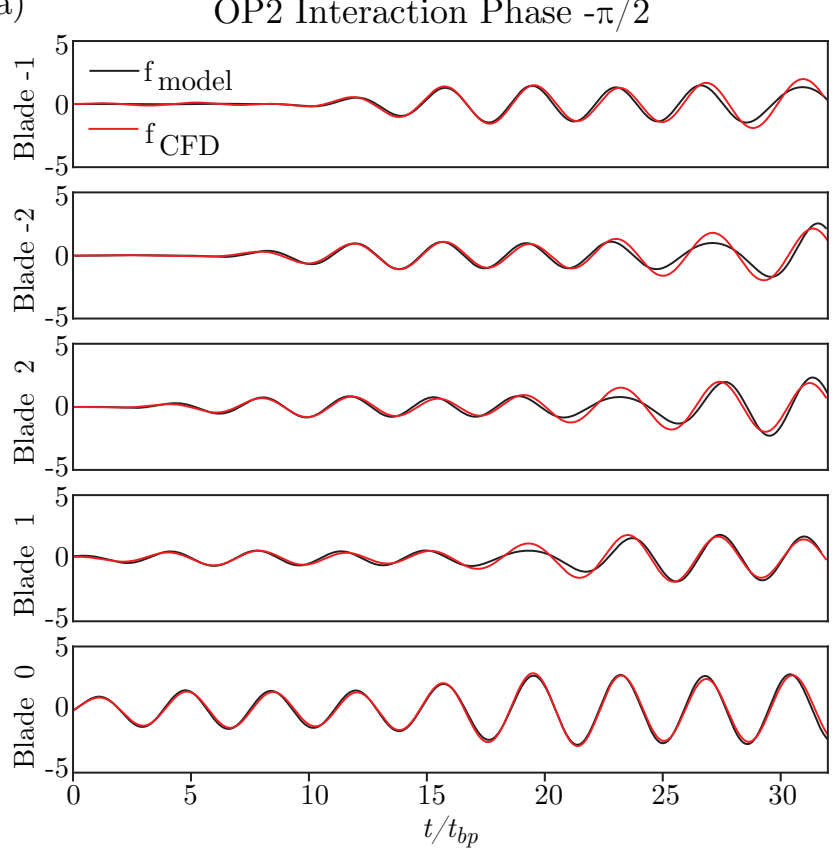

b)
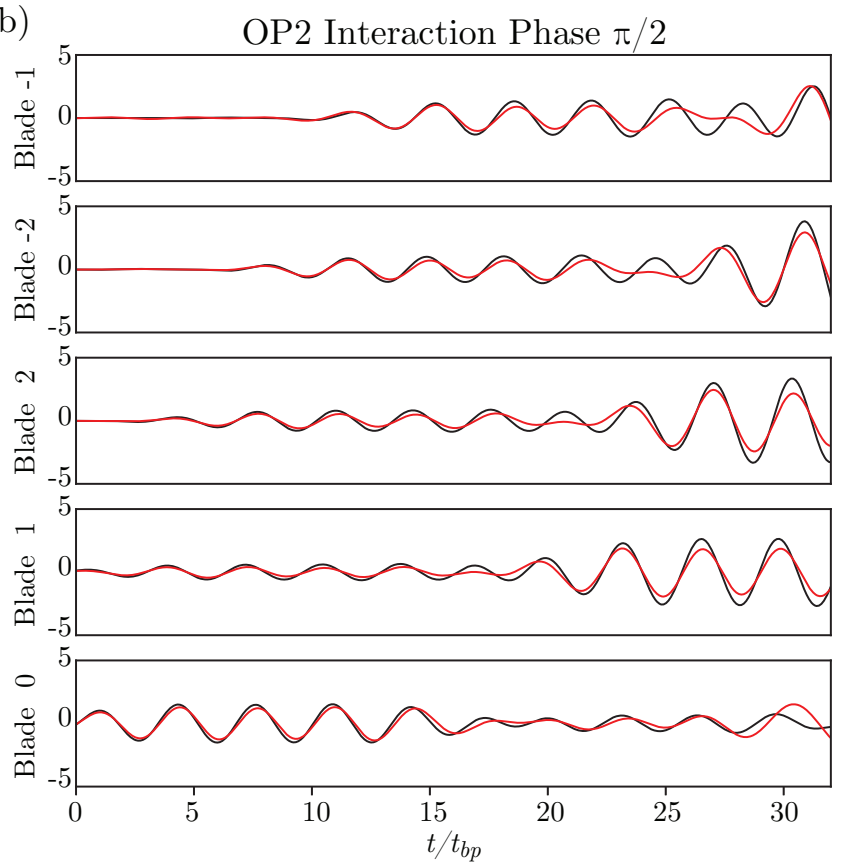

Figure 17: Comparison of blade forcing between CFD and semi-analytical model at OP2 for two interaction phases, ,a) $\varphi=-\pi / 2$, b) $\varphi=+\pi / 2$

Therefore, this section repeats the analysis for a range of propagation speeds, covering those measured in experiments and assuming that the tuned parameters $\left(L_{\theta}, L_{\Gamma}, C_{s}\right.$ etc.) remain constant which is of course not strictly true. The graphs presented in Fig. 20 summarize the results of this variation in $c_{\text {prop }}$. Fig. 20a shows how the locked-in aerodynamic wave number varies with propagation speed, while Fig. 20b shows the resonant structural nodal diameter, i.e. the aerodynamic wave number aliased on the 21-bladed rotor. The size of the

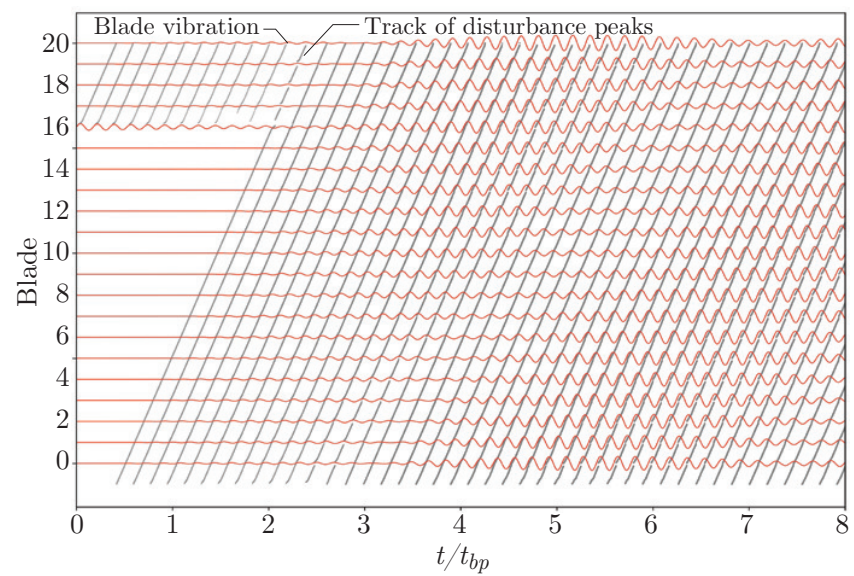

Figure 18: Disturbance evolution over 8 revolutions as predicted by semianalytical model for 21-bladed rotor. Initial disturbance at Blade $16: 1^{\circ}$, $\Omega_{a}^{S}=0.5 \Omega_{r}^{S}$.
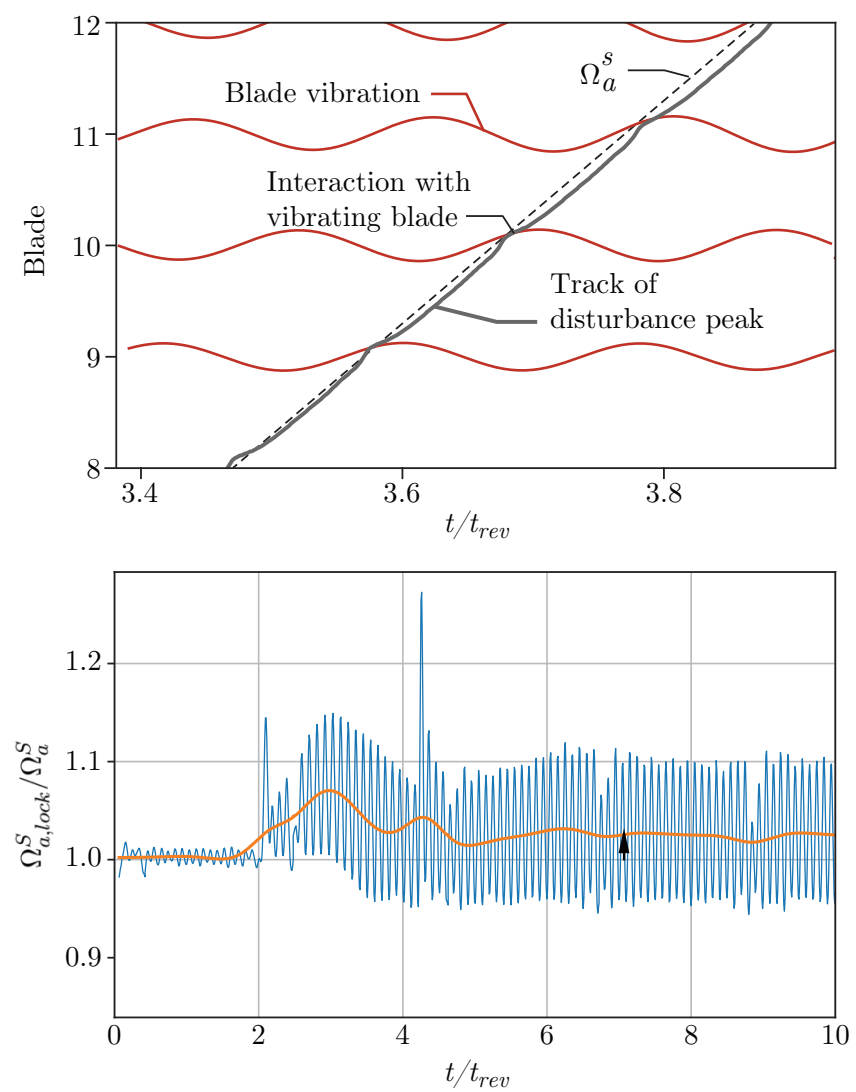

Figure 19: Change in propagation speed as predicted by semi-analytical model for 21-bladed rotor 


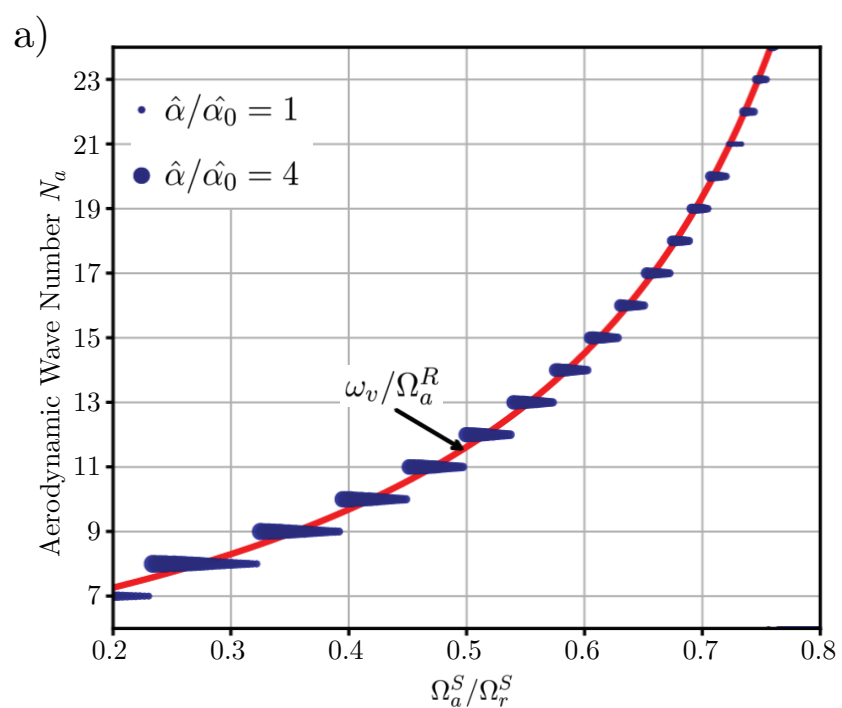

b)

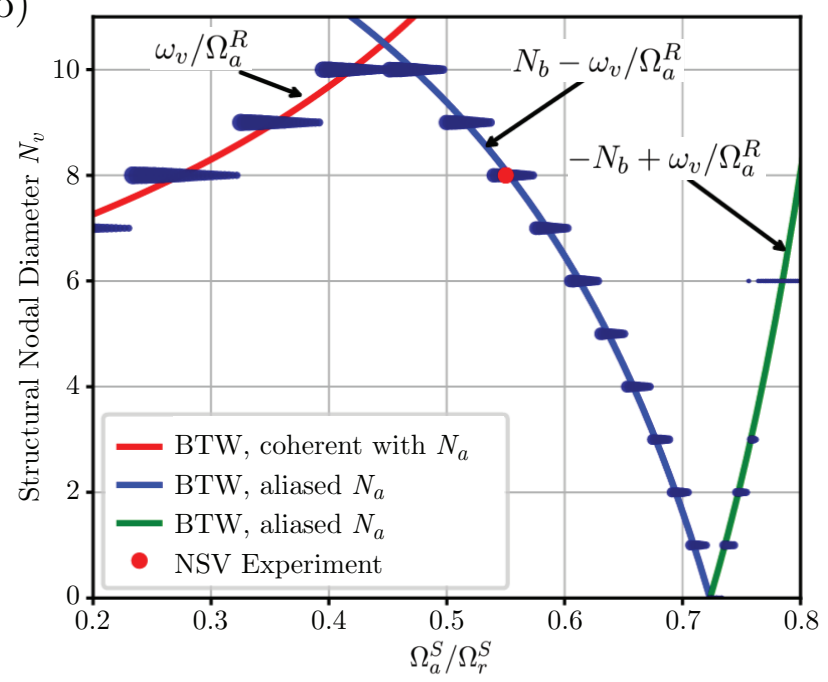

Figure 20: Predicted aerodynamic wave number (a) and excited structural nodal diameter pattern (b) for a range of free propagation speeds. Forward travelling (FTW) and backward travelling (BTW) waves as a result of aliasing on 21-bladed rotor. The experimental vibration mode is indicated. Symbol size represents average blade vibration amplitude $\hat{\alpha}$ after 20 revolutions

markers indicate the average vibration amplitude after periodic convergence.

As expected, the system always locks in to the nearest possible structural nodal diameter, which is either equal to the aerodynamic wave number or aliases if the aerodynamic wave number exceeds $0.5 N_{b}$. The points where the lines of $\omega_{v} / \Omega_{a}^{R}$ crosses integer wave numbers present a resonance condition where the free propagation speed, aerodynamic wave number and vibration frequency match without velocity adjustment $\left(\Omega_{a}^{R}=\Omega_{a, \text { lock }}^{R}\right)$. In between these, an adjustment of the global velocity as seen above and Section 6.3 is necessary. If the global propagation speed is below the resonant speed (to the left of the line) the oscillation amplitude is higher than when it is above the resonant speed.

For the rotor under investigation, propagation speeds be- tween $\Omega_{a}^{S}=0.5 \Omega_{r}^{S}$ and $0.8 \Omega_{r}^{S}$ were recorded depending on the operating point and VIGV setting. At these speeds, only forward travelling structural waves (FTW), turning against the direction of the aerodynamic disturbance, are susceptible to resonance with the aerodynamic disturbance. In the published experiment $N_{v}=8, N_{a}=13$ and $\Omega_{a}^{S}=0.55 \Omega_{r}^{S}$ were measured [16]. The model predicts this vibration mode. The range of $\Omega_{a}^{S}$ for Nodal Diameter $N_{v}=8$ extends from approximately 0.53 to $0.57 \Omega_{r}^{S}$. The experiments were also repeated at partspeed conditions with closed VIGVs, and the propagation speed was found to be $0.52 \Omega_{r}^{S}$ with Nodal Diameter $N_{v}=6$. This mode was also successfully predicted by the model without further tuning (plots omitted for brevity).

For higher propagation speeds, the adjustment range of propagation speed narrows for each nodal diameter. This means that a natural fluctuation of $\Omega_{a}^{S}$ leads to the simultaneous excitation of multiple structural waveforms and hence prevents the development of a singular dominant mode.

The results show that the highest amplitudes are achieved if the locked-in propagation speed is higher than the free propagation speed, which is coherent with experimental measurements [16].

Although the presented parameter variation of $\Omega_{a}^{S}$ exceeds the technically relevant range and the assumption of constant parameters is bold, such analysis aids understanding of the NSV mechanism and was shown to successfully identify critical modes and operating points.

\section{Description of NSV mechanism and comparison to other aeroelastic phenomena}

Following the insights gained from the CFD and the semianalytical model, the cause and evolution of near-stall NSV in axial compressors can be explained as follows: When a blade is subjected to a small change in conditions it creates a vorticity disturbance, which either originates from boundary layer separation or, as in the present case, from a fluctuation of the shear layer between the tip leakage and main passage flow. When operating very close to stall, the vorticity is not convected through the blade passage and out of the rotor domain but circumferentially towards the adjacent blade. The free propagation speed of the disturbance is determined by the mean flow. When the vorticity disturbance arrives at a trailing blade it induces changes in the vorticity generation on the blade surface, which in turn produces a subsequent disturbance. ${ }^{1}$

Once the aerodynamic disturbance interacts with a vibrating blade, the interaction modulates the phase of the disturbance. This effect depends on the phase of the vibration at time of arrival: the blade can either 'hold' the incoming vorticity for a certain time or shed it immediately. In the (numerical and experimental) measurements, this manifests itself in an apparent change in global propagation velocity. This shift of

${ }^{1}$ Note that, due to the use of a fixed control volume to quantify the disturbance, the terms circulation and vorticity are interchangeable in the context of this description. 
propagation speed is the mechanism through which lock-in occurs. Through an adjustment of the global velocity, the disturbance becomes phase-locked with the vibration and a coherent aerodynamic pattern with a fixed aerodynamic wave number is formed. This can be regarded as a lock-in of structural and aerodynamic nodal diameter patterns. The range, within which the local velocity can adjust, is bound. Beyond this limit, a different nodal diameter pattern is selected which leads to the 'stair case' plots seen in Fig. 20 and in acceleration/deceleration manoeuvres in test rigs [9].

The CFD results have shown that NSV can occur in a regime where the relationship between forcing and oscillation amplitude is still linear, and that flow separation is not strictly necessary. Multiple trigger mechanisms are therefore possible. An aerodynamic unsteadiness independent of blade vibration normally exists. This was seen in experiments as well as equivalent full domain CFD simulations [16], [? ]. Such an aerodynamic unsteadiness may cause local vortex shedding which will lead to NSV if the amplitude is large enough to cause blade deflection and/or induce further shedding on the trailing blade.

The propagation of the disturbance is very sensitive to operation point. At a point very close to stall, when the passage is blocked, the disturbance propagates circumferentially with little attenuation. At a slightly higher mass flow, on the other hand, the forcing of remote blades decreases with distance from the oscillating blade.

The excitation seen by the blades during this process depends on the amplitude of the disturbance, the modal force's sensitivity to a change in lift and the modal stiffness of the blade. In Eqn. 12 we saw that the forcing amplitude scales linearly with the term $\frac{1}{2} c_{1} \rho v_{\infty}^{2} c \bar{L}_{\theta}$ where $c_{1}$ is inversely proportional to modal mass $m_{r}=\Phi^{t} M \Phi$. This explains why NSV is significantly more common in modern high-speed and light-weight compressors. The lack of mechanical damping in blisks further aggravates the problem.

The NSV mechanisms described above bear some resemblance to other aeroelastic phenomena. Since an aerodynamic unsteadiness exists before blade vibration, the problem could initially be considered an non-synchronous forced response problem. However, with blade vibration the forcing function is modified such that a decoupled forced response analysis is not valid. The fact that the instability relies on blade vibration then points towards a flutter phenomenon. However, the mechanism is distinctly different from classical, linear flutter because the aerodynamic disturbance (whether generated with or without blade vibration) does not decay sufficiently circumferentially. Before lock-in two frequencies, one governed by blade vibration and the other by the circumferential propagation velocity, exist. In this sense, the NSV mechanisms resembles buffeting where an aerodynamic instability locks in with blade vibration.

Although a rigorous classification is difficult at this stage, the results have clearly demonstrated that this type of NSV is a convective phenomenon and that acoustic effects are negligible. The authors therefore propose the term 'convective NSV' to differentiate it from acoustically driven aeroelastic instabilities in the future. The term 'stall' should be avoided in this context because the blade boundary layer does not separate and the operating point does not drop significantly.

\section{Conclusion}

This study presented an explanation for the previously poorly understood phenomenon of non-synchronous vibrations in axial compressors. Evidence for the lock-in between convected aerodynamic disturbances and a structural vibration pattern during NSV was obtained from validated CFD computations. The physical mechanisms driving NSV and the lock-in process were identified through a parametric study. The study proved that this type of NSV is dominated by a convective phenomenon.

Using these findings, a semi-analytical model based on single degree of freedom oscillators was developed. The model not only correctly reproduced the results from the reduceddomain computations, but also predicted the experimental vibration modes at two different speeds. It can therefore serve to assess the sensitivity of NSV to driving parameters, and to identify critical interaction modes for any geometry. When calibrated with reduced-domain simulations, it is also capable of quantitative predictions. To the authors knowledge, this is the first time this has been achieved with a computationally inexpensive, semi-analytical model.

In addition to the identification of critical operating conditions and modes, the model also allows the assessment of NSV mitigation measures, such as structural mistuning, which is well known to reduce vibration amplitudes $[33,34]$, at an early stage in the design.

\section{Acknowledgements}

The authors are grateful to Professor Cumpsty of Imperial College for many useful discussions and generous advice, and Professor Greitzer of MIT for insightful comments, particularly on the nature of the aerodynamic disturbance. The authors also thank their colleagues Thomas Bontemps and Xavier Ottavy for their input.

This research was supported by Rolls-Royce plc and the EPSRC under the Prosperity Partnership Grant "Cornerstone: Mechanical Engineering Science to Enable Aero Propulsion Futures", Grant Ref: EP/R004951/1.

\section{References}

[1] R. Parker, Acoustic resonances and blade vibration in axial flow compressors, J. Sound Vib. 92 (4) (1984) 529 - 539. URL https : //doi . org/10.1016/0022-460X (84)90196-2

[2] R. E. Kielb, J. W. Barter, J. P. Thomas, K. C. Hall, Blade excitation by aerodynamic instabilities: A compressor blade study, in: ASME Turbo Expo 2003, 2003, pp. 399-406. URL https : //doi .org/10.1115/GT2003-38634

[3] H. D. Vo, Role of tip clearance flow in rotating instabilities and nonsynchronous vibrations, J. Propul. Power 26 (3) (2010) 556-561. URL https://doi .org/10.2514/1.26709

[4] J. Thomassin, H. D. Vo, N. W. Mureithi, The tip clearance flow resonance behind axial compressor nonsynchronous vibration, J. Turbomach. 133 (4) (2011) 041030.

URL https ://doi.org/10.1115/1.4001368 
[5] S. T. Clark, R. E. Kielb, K. C. Hall, Developing a reduced-order model to understand non-synchronous vibration (NSV) in turbomachinery, in: ASME Turbo Expo 2012, American Society of Mechanical Engineers, 2012, pp. 1373-1382.

URL https ://doi.org/10.1115/GT2014-25547

[6] D. Möller, M. Jüngst, F. Holzinger, C. Brandstetter, H.-P. Schiffer, S. Leichtfuß, Mechanism of nonsynchronous blade vibration in a transonic compressor rig, J. Turbomach. 139 (1) (2017) 011002. URL https ://doi .org/10.1115/1.4034029

[7] M. Drolet, H. D. Vo, N. W. Mureithi, Effect of tip clearance on the prediction of nonsynchronous vibrations in axial compressors, J. Turbomach. 135 (1) (2013) 011023.

URL https://doi.org/10.1115/1.4006401

[8] H.-S. Im, G.-C. Zha, Simulation of non-synchronous blade vibration of an axial compressor using a fully coupled fluid/structure interaction, in: ASME Turbo Expo 2012, American Society of Mechanical Engineers, 2012, pp. 1395-1407.

URL https : //doi .org/10.1115/GT2012-68150

[9] J. Dodds, M. Vahdati, Rotating stall observations in a high speed compressor-part ii: Numerical study, J. Turbomach. 137 (5) (2015) 51003-51010.

URL https : //doi .org/10.1115/1.4028558

[10] A. L. Fiquet, C. Brandstetter, S. Aubert, M. Philit, Non-synchronous aeroacoustic interaction in an axial multi-stage compressor, J. Turbomach. (2019) 1-18.

URL https ://doi.org/10.1115/1.4044675

[11] R. E. Kielb, K. C. Hall, M. Spiker, J. P. Thomas, E. T. Pratt Jr, R. Jeffries, Non-synchronous vibration of turbomachinery airfoils, Tech. rep., Duke University, Durham NC (2006).

URL http://people.duke.edu/ jthomas/papers/papers/ hcfnsv2004.pdf

[12] L. di Mare, M. Vadati, B. Mueck, N. H. Smith, N. Birch, Aeroelastic instability of fan blades at high altitudes, in: ASME Turbo Expo 2009, American Society of Mechanical Engineers, 2009, pp. 1765-1772.

URL https://doi.org/10.1115/GT2009-60091

[13] H. Emmons, Compressor surge and stall propagation, Trans. of the ASME 77 (4) (1955) 455-467.

[14] I. Day, N. Cumpsty, The measurement and interpretation of flow within rotating stall cells in axial compressors, J. Mech. Eng. Sci. 20 (2) (1978) $101-114$.

URL https : //doi.org/10.1243/JMES_JOUR_1978_020_017_02

[15] N. Cumpsty, E. M. Greitzer, A simple model for compressor stall cell propagation', J. Eng. Power 104 (1) (1982) 170-176. URL https : //doi .org/10.1115/1.3227246

[16] C. Brandstetter, M. Juengst, H.-P. Schiffer, Measurements of Radial Vortices, Spill Forward, and Vortex Breakdown in a Transonic Compressor, J. Turbomach. 140 (6) (2018) 061004-061004-14. URL https : //doi .org/10.1115/1.4039053

[17] N. Cumpsty, Discussion: Rotating instabilities in an axial compressor originating from the fluctuating blade tip vortex ( Mailach, R., Lehmann, I., and Vogeler, K., 2001, J. Turbomach., 123, no. 3, pp. 453-460), J. Turbomach. 123 (2001) 461-461. URL http://dx.doi.org/10.1115/1.1371008

[18] F. Kameier, W. Neise, Rotating blade flow instability as a source of noise in axial turbomachines, J. Sound Vib. 203 (5) (1997) 833-853. URL https://doi.org/10.1006/jsvi.1997.0902

[19] R. Mailach, I. Lehmann, K. Vogeler, Rotating Instabilities in an Axial Compressor Originating From the Fluctuating Blade Tip Vortex, J. Turbomach. 123 (3) (2001) 453-460. URL https : //doi .org/10.1115/1.1370160

[20] H. D. Vo, C. S. Tan, E. M. Greitzer, Criteria for spike initiated rotating stall, J. Turbomach. 130 (1) (2008) 11023-11029.

URL https : //doi.org/10.1115/1.2750674

[21] G. Pullan, A. M. Young, I. J. Day, E. M. Greitzer, Z. S. Spakovszky, Origins and structure of spike-type rotating stall, J. Turbomach. 137 (5) (2015) 51007-51011.

URL https : //doi .org/10.1115/1.4028494

[22] M. Eck, S. Geist, D. Peitsch, Physics of prestall propagating disturbances in axial compressors and their potential as a stall warning indicator, Applied Sciences 7 (3) (2017) 285.

URL https : //doi .org/10.3390/app7030285
[23] C. Hah, J. Bergner, H.-P. Schiffer, Tip clearance vortex oscillation, vortex shedding and rotating instabilities in an axial transonic compressor rotor, in: ASME Turbo Expo 2008, American Society of Mechanical Engineers, 2008, pp. 57-65. URL https://doi.org/10.1115/GT2008-50105

[24] M. Inoue, M. Kuroumaru, T. Tanino, M. Furukawa, Propagation of multiple short-length-scale stall cells in an axial compressor rotor, J. Turbomach. 122 (1) (2000) 45-54. URL https://doi.org/10.1115/1.555426

[25] A. Young, I. Day, G. Pullan, Stall warning by blade pressure signature analysis, J. Turbomach. 135 (1) (2013) 11033. URL https ://doi .org/10.1115/1.4006426

[26] C. Brandstetter, C. Biela, M. Kegalj, H.-P. Schiffer, PIV-measurements in a transonic compressor test rig with variable inlet guide vanes, in: Proceedings of 20th ISABE 2011, Gothenburg, Sweden, 2011, pp. 448-458. URL http://tubiblio.ulb.tu-darmstadt.de/74497/

[27] M. Vahdati, A. I. Sayma, M. Imregun, G. Simpson, Core-compressor rotating stall simulation with a multi-bladerow model, in: K. C. Hall, R. E. Kielb, J. P. Thomas (Eds.), Unsteady Aerodynamics, Aeroacoustics and Aeroelasticity of Turbomachines, Springer Netherlands, Dordrecht, 2006, pp. 313-329.

URL https : //doi .org/10.1007/1-4020-4605-7-23

[28] E. Greitzer, C. Tan, M. Graf, Internal flow: Concepts and applications. 2004 (2004).

URL https ://doi.org/10.1017/CB09780511616709

[29] L. Graftieaux, M. Michard, N. Grosjean, Combining PIV, POD and vortex identification algorithms for the study of unsteady turbulent swirling flows, Measurement Science and Technology 12 (9) (2001) 1422.

URL http: //stacks . iop.org/0957-0233/12/i=9/a=307

[30] M. Namba, H. Nakagawa, A. Kubo, Lifting surface theory to predict aerodynamic forces induced by oscillating blades under interaction of three bladerows, J. Sound Vib. 326 (3) (2009) $599-622$. URL https : //doi.org/10.1016/j.jsv.2009.06.002

[31] D. M. Feiner, J. Griffin, A fundamental model of mistuning for a single family of modes, J. Turbomach. 124 (4) (2002) 597-605. URL https ://doi.org/10.1115/1.1508384

[32] T. Theodorsen, General theory of aerodynamic instability and the mechanism of flutter, NACA Technical Report 496 (1949). URL https : //ntrs . nasa.gov/search. jsp?R=19930090935

[33] E. Capiez-Lernout, C. Soize, M. Mbaye, Mistuning analysis and uncertainty quantification of an industrial bladed disk with geometrical nonlinearity, J. Sound Vib. 356 (2015) $124-143$. URL https://doi.org/10.1016/j.jsv.2015.07.006

[34] C. Martel, J. J. Sánchez-Álvarez, Intentional mistuning effect in the forced response of rotors with aerodynamic damping, J. Sound Vib. 433 (2018) $212-229$.

URL https://doi.org/10.1016/j.jsv.2018.07.020 